\title{
Comparative magnetotail flapping: an overview of selected events at Earth, Jupiter and Saturn
}

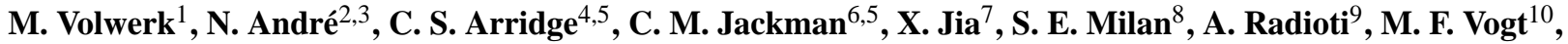 \\ A. P. Walsh ${ }^{4, *}$, R. Nakamura ${ }^{1}$, A. Masters ${ }^{11}$, and C. Forsyth ${ }^{4}$ \\ ${ }^{1}$ Space Research Institute, Austrian Academy of Sciences, Graz, Austria \\ ${ }^{2}$ Centre d'Étude Spatiale des Rayonnements, Université de Toulouse, UPS, Toulouse, France \\ ${ }^{3}$ CNRS, UMR 5187, Toulouse, France \\ ${ }^{4}$ Mullard Space Science Laboratory, University College London, Holmbury St. Mary, UK \\ ${ }^{5}$ Centre for Planetary Sciences at UCL/Birkbeck, London, WC1E 6BT, UK \\ ${ }^{6}$ Department of Physics and Astronomy, University College London, Gower Place, London, WC1E 6BT, UK \\ ${ }^{7}$ Dept. of Atmospheric, Oceanic and Space Sciences, University of Michigan, Ann Arbor, USA \\ ${ }^{8}$ Department of Physics and Astronomy, University of Leicester, Leicester, UK \\ ${ }^{9}$ Laboratoire de Physique Atmosphérique et Planétaire, Institut d'Astrophysique et de Géophysique, Université de Liège, \\ Liège, Belgium \\ ${ }^{10}$ Department of Physics and Astronomy, University of Leicester, Leicester, UK \\ ${ }^{11}$ Institute of Space and Astronautical Science, JAXA, Sagamihara, Japan \\ *now at: Science and Robotic Exploration Directorate, ESA/ESTEC, Noordwijk, the Netherlands
}

Correspondence to: M. Volwerk (martin.volwerk@oeaw.ac.at)

Received: 14 August 2012 - Revised: 22 March 2013 - Accepted: 12 April 2013 - Published: 6 May 2013

\begin{abstract}
A comparison of magnetotail flapping (the upand-down wavy motion) between the Earth and the two giant planets Jupiter and Saturn has been performed through investigation of the current sheet normal of the magnetotail. Magnetotail flapping is commonly observed in the Earth's magnetotail. Due to single spacecraft missions at the giant planets, the normal is determined through minimum variance analysis of magnetometer data during multiple intervals when the spacecraft crossed through the current sheet. It is shown that indeed a case can be made that magnetotail flapping also occurs at Jupiter and Saturn. Calculations of the wave period using generic magnetotail models show that the observed periods are much shorter than their theoretical estimates, and that this discrepancy can be caused by unknown input parameters for the tail models (e.g., current sheet thickness) and by possible Doppler shifting of the waves in the spacecraft frame through the fast rotation of the giant planets.
\end{abstract}

Keywords. Magnetospheric physics (Magnetotail; MHD waves and instabilities)

\section{Introduction}

Magnetotail flapping, the up-and-down wavy motion of the current sheet is now a well-established phenomenon in the Earth's magnetotail (see e.g., Sergeev et al., 1998; Zhang et al., 2002; Sergeev et al., 2003; Runov et al., 2005), identified in spacecraft data as multiple crossings of the current sheet. It has been shown that the waves, with periods of several minutes travel from the centre of the magnetotail to the flanks and that storm and substorm activity can excite and constrain the motion of the current sheet (Davey et al., 2012). During the passage of these waves the current sheet can be very strongly tilted in the YZ-plane by these flapping waves. Similar observations of multiple current sheet crossings have been presented from Jupiter (Israelevich and Ershkovich, 2006; Israelevich et al., 2007), but until now there have been no reported observations of this phenomenon at Saturn.

Early studies of the terrestrial magnetotail reported multiple current and neutral sheet crossings (neutral sheet defined as $B_{\mathrm{x}}=0$ ) observed by spacecraft (Speiser and Ness, 1967; 
Toichi and Miyazaki, 1976; Lui et al., 1978). Although various ideas were suggested to account for these observations, it was generally considered that these observations were due to the current sheet flapping in an up-and-down motion over the spacecraft. The multi-spacecraft Cluster mission later confirmed this by unambiguously determining the local velocity of the current sheet (Zhang et al., 2002; Sergeev et al., 2004). These observations presented a new view of the current sheet.

Cluster's unique view of the current sheet offered new insights into current sheet flapping. Zhang et al. (2002) showed that during the flapping the current sheet gets very warped (see their Figs. 3 and 5 and Fig. 4 in this paper), and the normal of the current sheet for the neutral sheet crossings was often tilted away from the $Z_{\mathrm{GSM}}$-axis over more than $90^{\circ}$ in the $Y Z_{\mathrm{GSM}}$-plane. Sergeev et al. (2004) performed a statistical study of magnetotail flapping events in 2001 using Cluster. Using timing analysis (Harvey, 1998) they found that the normal direction of the current sheet in the GSM XY-plane was always pointing away from the midnight meridian, indicating that the waves are travelling towards the flanks. Typically the y-component of the normal was largest and the average propagation velocity of the waves was $57 \mathrm{~km} \mathrm{~s}^{-1}$ for more quiet current sheets and $145 \mathrm{~km} \mathrm{~s}^{-1}$ for active ones.

The studies of current sheet flapping discussed above presented observations from a narrow range of distances along the tail for each individual study, either from a single spacecraft location or relatively closely separated (i.e., $\sim 1000 \mathrm{~km}$ ) multi-spacecraft observations by Cluster. On 5 August 2004 both Cluster and Double Star TC1, which were separated by $\sim 5 R_{\mathrm{E}}$ observed a flapping magnetotail. Using the four spacecraft Cluster data, timing analysis (Harvey, 1998) was performed on the $B_{\mathrm{x}}=0$ crossings to obtain the normal direction of the current sheet. This timing analysis showed that the current sheet was again very warped (see Fig. 3 in Zhang et al., 2005) during the flapping, with an oscillation period of $\sim 10 \mathrm{~min}$. The time difference between the Cluster spacecraft and TC1 showed that the flapping of the tail takes place over at least a distance of $5 R_{\mathrm{E}}$ along the tail, and that the waves are travelling at a slight angle with respect to the GSM Y-direction (Volwerk et al., 2005).

Theoretical models have investigated those inherent instabilities of the Earth's magnetotail that are driven by the magnetic field and plasma gradients along and across the tail. These models delivered the dispersion relation for these types of large-scale waves in the Earth's magnetotail and have been presented by Golovchanskaya and Maltsev (2005) and Erkaev et al. (2008, 2010). Lately, Forsyth et al. (2009) showed that the so-called "double gradient model" in which the flapping waves arise through the combination of the magnetic field gradients $\partial B_{\mathrm{x}} / \partial z$ and $\partial B_{\mathrm{Z}} / \partial x$, as presented by Erkaev et al. (2008), best fit the observations made by Cluster of a flapping current sheet in 2001.

The flapping motion of the current sheet has enabled detailed studies of the vertical structure of the current sheet to be made, given that the observing spacecraft can pass through the current sheet on relatively short timescales. Sergeev et al. (1998) used three months of magnetic field and plasma data from the AMPTE/IRM satellite, to study the flapping motion of the Earth's magnetotail and the structure of the current sheet. They found that there was a correlation to the plasma velocity perpendicular to the plane of the current sheet $\left(V_{\mathrm{Z}, \mathrm{GSM}}\right)$ and the $d B_{\mathrm{X}, \mathrm{GSM}} / d t$. A linear regression was made between the velocity and the magnetic field time derivative:

$V_{\mathrm{z}}=A_{0}+A_{1} \frac{d B_{\mathrm{x}}}{d t}$.

For the cases in which a strong correlation was found, an estimate for the scale height $h$ of the current sheet could be obtained when a planar current sheet is assumed (which holds when $B_{\mathrm{Z}} \ll B_{\mathrm{x}}$ ):

$h=\frac{B_{\mathrm{L}} A_{1}}{\sqrt{1+k_{1}^{2}}}$,

where $k_{1}$ is the regression coefficient between $B_{\mathrm{y}}$ and $B_{\mathrm{x}}$, and $B_{\mathrm{L}}$ is the lobe magnetic field. This leads to current density estimate of:

$j \approx \frac{800}{\left(A_{1}\left(1+k_{1}\right)\right)}$,

with the current in $\mathrm{nA} / \mathrm{m}^{2}$. From the AMPTE/IRM data it was concluded that during flapping events the current density was more intense $\left(j \sim 10-20 \mathrm{nA} \mathrm{m}^{-2}\right)$ and the current sheet thinner $\left(h \sim 0.2-1 R_{\mathrm{E}}\right)$ than was expected from magnetospheric models for quiescent magnetotails $\left(j \leq 5 \mathrm{nA} \mathrm{m}^{-2}\right.$, $h \geq 1 R_{\mathrm{E}}$, see e.g., Zhang et al., 2006). A recent statistical study by Davey et al. (2012) showed observations of magnetotail flapping events also have a similar occurrence distribution across the magnetotail to fast magnetospheric flows, suggesting a link with substorm activity.

At Jupiter some investigations on "flapping" current sheets were done by Israelevich and Ershkovich (2006) and Israelevich et al. (2007) using multiple Voyager 2 and Galileo crossings of the Jovian current sheet in order to find if its structure is Harris-like or bifurcated. In a Harris current sheet (Harris, 1962) between two regions of oppositely directed magnetic fields, there is pressure balance between the plasma and the magnetic field and the magnetic field can be described by a hyperbolic tangent with a specific scale height $H$ :

$B_{\mathrm{x}}(z)=B_{0} \tanh \left\{\frac{z}{H}\right\}$.

This model has maximum current density in the centre of the current sheet described by:

$J_{\mathrm{y}}(z)=\frac{B_{0}}{H} \operatorname{sech}^{2}\left\{\frac{z}{H}\right\}$.

In contrast to a Harris sheet the current sheet can also be bifurcated, which means that there is a local minimum in the 
current density at $z=0$ which is flanked by two maxima at $z= \pm z_{0}$ (see e.g., Hesse and Schindler, 1986; Runov et al., 2003).

It should be understood that "flapping" in Israelevich and Ershkovich (2006) and Israelevich et al. (2007) refers to multiple crossings of $B_{\mathrm{x}}=0$, but these crossings need not show the characteristics of the Earth's magnetotail flapping. These papers showed a number of profiles of $\partial B_{X} / \partial t$ against $B_{\mathrm{x}}$, taken to be indicative of the current density profiles of the current sheets. Their observations included 14 bifurcated sheets within the period from 13 September 1996 until 26 December 1998. They concluded that bifurcation is not a common feature of the Jovian current sheet. When present, the bifurcation is possibly generated by the ion pressure anisotropy in the plane perpendicular to the magnetic field. They present a model for the Jovian current sheet using Vlasov and Maxwell's equations and introduce a strong anisotropy in the ion pressure. They obtain a current density for the Jovian current sheet depending on the vector potential $A(z)$ of the magnetic field (with $B=d A / d z$ ):

$\tilde{j}=\sqrt{\tilde{A}_{0}^{2}-\ln \left(1-\tilde{B}^{2}\right)} \exp \left\{\tilde{A}_{0}^{2}-\ln \left(1-\tilde{B}^{2}\right)\right\}$.

where, $\tilde{A}, \tilde{B}$ are the dimensionless vector potential and magnetic field. The profile of this Jovian current density fits well with that of the Earth for bifurcated current sheets.

In the Kronian magnetotail there are various periodicities related to the rotational period of Saturn, as discussed in Arridge et al. (2011); Andrews et al. (2012); Provan et al. (2012). It was found that the current sheet in the Kronian magnetosphere is hinged, similar to those at the Earth and Jupiter, and that it is located above the rotational equator and adopts a bowl shape over the midnight-dawn-noon local time sectors (Arridge et al., 2008).

Cassini data have also shown that reconnection happens in Saturn's magnetotail (see e.g., Jackman et al., 2007, 2008; Masters et al., 2011), showing the presence of plasmoids (Hones et al., 1984) and return flows, and indicating that the recurrence rate of these events may be in the region of $\sim 2.4$ days (Jackman et al., 2011). However, there have not been any studies with respect to possible magnetotail flapping as have been done at Earth and Jupiter.

In this paper, we try to find whether the magnetotails of Jupiter and Saturn show a similar flapping behaviour as the Earth's magnetotail, which was not done in the Jupiter papers discussed above. There is strong evidence that substormlike processes occur in the magnetotails of the giant planets, where stored energy is released through reconnection events as shown by e.g., Jackman et al. (2007); Masters et al. (2011); Vogt et al. (2010), which may then be able to drive the flapping motion of the magnetotail. However, for the six events that we will be investigating in this paper at these two giant planets plasma velocity vectors are not routinely available. Therefore, we first examine the characteristics of the flapping motions at the Earth using magnetometer data from Cluster and Double Star in Sect. 2.1. These results are then compared with data from Cassini at Saturn in Sect. 2.2 and Galileo at Jupiter in Sect. 2.3. A comparison with theoretical models is presented in Sect. 3, the possible current sheet bifurcation is discussed in Sect. 4, and we end with a discussion in Sect. 5.

In this paper, at the Earth, we will use the Geocentric Solar Magnetospheric system (GSM) which has its $\mathrm{x}$-axis from the Earth to the Sun. The y-axis is defined to be perpendicular to the Earth's magnetic dipole so that the XZ-plane contains the dipole axis. The positive $\mathrm{z}$-axis is chosen to be in the same sense as the northern magnetic pole. This is the preferred coordinate system for the Earth's magnetotail, as e.g., for magnetotail flapping the rocking of the current sheet normal only displays its characteristic motion in this system. Therefore, at the giant planets we will use the planetocentric Solar Magnetospheric (pSM) coordinate system which is similarly defined with the specific planet as the origin.

\section{Magnetotail flapping at Earth, Saturn and Jupiter}

In Fig. 1 we show the relative location within their respective magnetospheres of all events discussed in this paper. The event from Cluster is labelled $\mathrm{Cl}$, the events from Jupiter are labelled 141, 171 and 173 and the events from Saturn are labelled 144, 249 and 265. Also shown are the approximate locations of the magnetopause for the Earth (polynomial values in Table 2 of Fairfield, 1971), Jupiter (Joy et al., 2002, with solar wind dynamic pressure $P_{\text {dyn }} \approx 0.2 \mathrm{nPa}$ ) and Saturn (Arridge et al., 2006, with $P_{\text {dyn }} \approx 0.01 \mathrm{nPa}$ ) in planetary radii. This gives an impression of where the events roughly take place inside the planetary magnetosphere.

In this paper, we will use data from the fluxgate magnetometer on Cluster (Balogh et al., 2001) from 2004, Cassini magnetic field data (Dougherty et al., 2004) at $1 \mathrm{~s}$ and $1 \mathrm{~min}$ resolution and the plasma data (Young et al., 2004) from 2006 and Galileo magnetometer data (Kivelson et al., 1992) at $27 \mathrm{~s}$ resolution from the G8 orbit of Galileo in 1997.

\subsection{Observations at Earth}

On 5 August 2004 both Cluster and Double Star TC1 observed a flapping magnetotail as discussed by Zhang et al. (2005) and Volwerk et al. (2005). In Fig. 2, the magnetic field components and magnitude are shown for the four (black, red, green, blue) Cluster spacecraft (Balogh et al., 2001) and (magenta) Double Star TC1 (Carr et al., 2005). The yellow shaded area in the figure shows the interval of magnetotail flapping.

Using the Cluster four-spacecraft data, timing analysis (Harvey, 1998) was performed on the $B_{\mathrm{x}}=0$ crossings to obtain the normal direction of the current sheet. The results of this timing analyses are shown in Table 1, and the quasi period for this current sheet oscillation is $\sim 10 \mathrm{~min}$. The time difference between the Cluster spacecraft and TC1 showed 


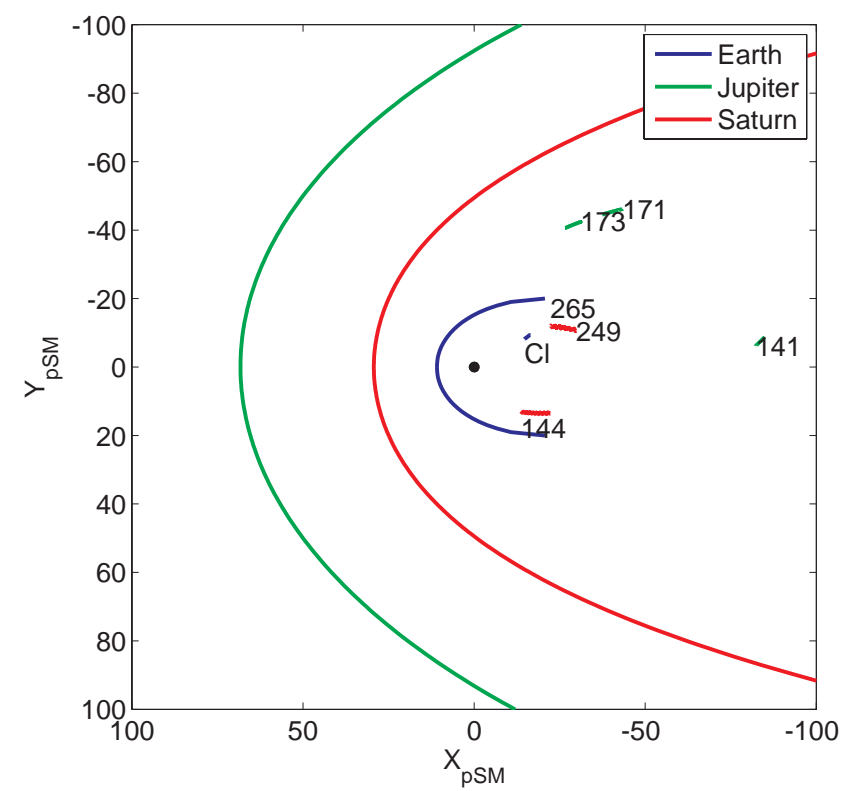

Fig. 1. The location of the flapping events in the planetary magnetotails. Shown are the magnetopauses of the Earth (blue), Jupiter (green) and Saturn (red) and the events in the same colours all in relative planetary radii in planetary Solar Magnetic (pSM) coordinates.

that the flapping of the tail takes place over at least a distance of $5 R_{\mathrm{E}}$ along the tail, and that the waves are travelling at a slight angle with respect to the $Y$-direction (see Zhang et al., 2005; Volwerk et al., 2005), a schematic view of which is shown in Fig. 3.

As the Galileo and Cassini missions at Jupiter and Saturn, respectively, are single spacecraft missions, timing analysis is not possible there. However, the normal of the current sheet can also be determined through Mininum Variance Analysis (MVA, Sonnerup and Cahill, 1967; Sonnerup and Scheible, 1998) over intervals that contain the $B_{\mathrm{x}}=0$ crossing. Also, the data resolution is lower at the outer planets for the events that we are looking at: for Galileo the data sampling rate is $24 \mathrm{~s}$, whereas for Cassini the sampling rate used here is $1 \mathrm{~min}$ (down sampled from $1 \mathrm{~s}$ ), compared to the $22 \mathrm{~Hz}$ data from Cluster. Because MVA gives better results on smoothed datasets, we have performed the MVA on spinresolution ( $4 \mathrm{~s}$, which is a higher resolution than will be used at Jupiter and Saturn) Cluster data and compare the results of the MVA normals with the timing normals in Table 1. In order to get an error estimate of the minimum variance direction we also calculate the error cone around the minimum variance direction, i.e., the angles of this cone in the intermediate and maximum variance directions (for the equations see, e.g., Sonnerup and Scheible, 1998).

For a wave travelling from the centre of the tail towards the flanks, one expects that the $\mathrm{x}$-component of the normal is minor and the $y$ - and z-components to oscillate between equal

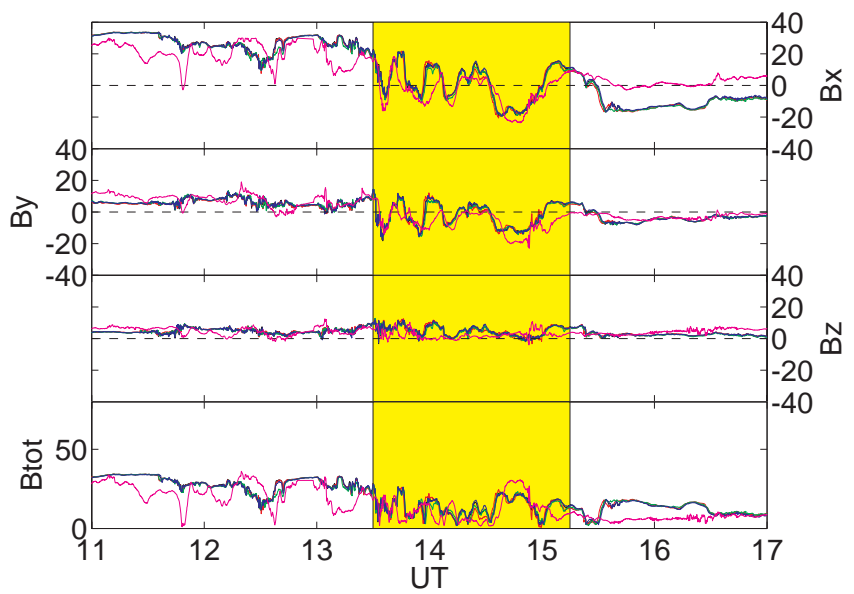

Fig. 2. The Cluster (black, red, green blue) and Double Star TC1 (magenta) magnetic field data for 5 August 2004. From top to bottom are shown the field components $B_{\mathrm{X}}, B_{\mathrm{y}}, B_{\mathrm{Z}}$ and the magnitude $B_{\text {tot }}$. The Cluster spacecraft were located $\sim 15 R_{\mathrm{E}}$ and TC1 $\sim 10 R_{\mathrm{E}}$ down the tail, both at a local time of $\sim 02: 00 \mathrm{LT}$. The multiple $B_{\mathrm{X}}=0$ crossings were identified as magnetotail flapping (Zhang et al., 2005).

signs for both components and opposite signs as shown in Sergeev et al. (2004). It is clear that the directions of the current sheet normal for both methods (timing and MVA) are not the same as listed in Table 1, however, the pattern of the yand z-components are quite similar with respect to the signs of both components, except for crossing \#5 for which the ratio of the intermediate and minimum eigenvalue is rather small, $\lambda_{\text {med }} / \lambda_{\text {min }}=3$. We note that in their comparison of MVA and timing analysis, Sergeev et al. (2006b) showed that the minimum variance direction of a flapping current sheet was within 20-30 degrees of the timing vector for values of $\lambda_{\text {med }} / \lambda_{\text {min }}>4$. A graphical interpretation of the current sheet normals in the YZ-plane is given in Fig. 4. Here we plot the normal direction as a solid arrow and the error cone around this direction, obtained from the MVA analysis, as dotted lines around the arrow. In the top part of Fig. 4 we have taken the timing normals and drawn an artist's impression of the warping of the current sheet as indicated by the normal directions in the YZ-plane. The current sheet should be at right angles to the arrows that represent the normals.

Although the absolute direction of the current sheet normal may not be correct using MVA on low-resolution data, as compared to multi-spacecraft timing analysis, its general behaviour is well captured and we can use this method to investigate possible magnetotail flapping at the giant planets.

\subsection{Observations at Saturn}

We use the Cassini magnetic field data (Dougherty et al., 2004) at 1 min resolution (down sampled from $1 \mathrm{~s}$ resolution) and when possible the plasma data (Young et al., 2004) for the year 2006, in which Cassini went far down the Kronian 
Table 1. Current sheet normals using timing analysis (Zhang et al., 2005) and MVA for 5 August 2004. Shown are the timing normal vector, the MVA normal vector, the ratio of minimum and intermediate eigenvalue, the error cone around the minimum variance direction in the intermediate and maximum variance directions $\Delta \phi_{12}$ and $\Delta \phi_{13}$ (in degrees).

\begin{tabular}{cc|ccc|cc}
\hline Crossing & Time & Normal & MVA & $\lambda_{\operatorname{med}} / \lambda_{\min }$ & $\Delta \phi_{12}$ & $\Delta \phi_{13}$ \\
\hline 1 & $13: 34$ & $(-0.283,-0.677,-0.679)$ & $(0.24,-0.26,-0.93)$ & 2 & 1.7 & 9.7 \\
2 & $13: 38$ & $(0.091,-0.895,-0.436)$ & $(0.32,-0.72,-0.60)$ & 8 & 0.6 & 1.7 \\
3 & $13: 47$ & $(0.128,-0.931,0.342)$ & $(0.50,-0.87,0.04)$ & 9 & 0.2 & 2.3 \\
4 & $13: 57$ & $(0.483,-0.624,-0.614)$ & $(0.67,-0.69,-0.24)$ & 10 & 0.2 & 2.3 \\
5 & $14: 07$ & $(0.545,-0.754,0.368)$ & $(0.63,-0.76,-0.01)$ & 3 & 0.6 & 2.3 \\
6 & $14: 14$ & $(0.205,-0.302,-0.931)$ & $(0.42,-0.60,-0.67)$ & 9 & 0.2 & 1.7 \\
7 & $14: 32$ & $(0.512,-0.742,0.433)$ & $(0.52,-0.85,0.01)$ & 3 & 0.2 & 2.9 \\
8 & $15: 00$ & $(0.509,-0.608,-0.610)$ & & & & \\
\hline
\end{tabular}

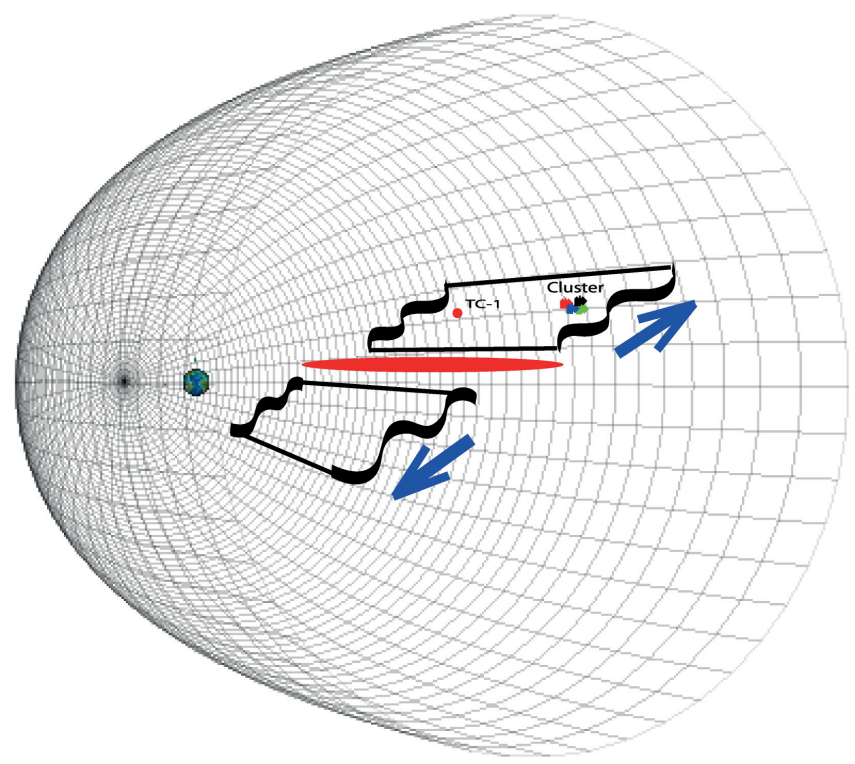

Fig. 3. A schematic view of the kink-like flapping wave emitted from the central part of the magnetotail in an equatorial view. This wave propagates towards the flanks of the magnetotail. Double Star TC- 1 and Cluster were located $\sim 5 R_{\mathrm{E}}$ apart at same local time and both observed this wave. The cone-shaped mesh represents the magnetopause.

tail and remained well within the centre of the tail, i.e., the orbit was located within the region of $-20 \leq Y_{\mathrm{KSM}} \leq 20$.

We have searched for half-hour intervals where there are multiple crossings of the current sheet and selected events for this case study on DOY 144, 249 and 265. Although the description of tail dynamics may be best described in the spherical KRTP coordinate system (see e.g., Jackman et al., 2009), in order to perform the analysis of the current sheet normal using MVA we need a Cartesian coordinate system. Also, we prefer to use the same coordinate system as at Earth, for reasons mentioned above, and for easier comparison between planets. The data have been transformed to the KSM (Kronian-Solar-Magnetospheric) coordi-

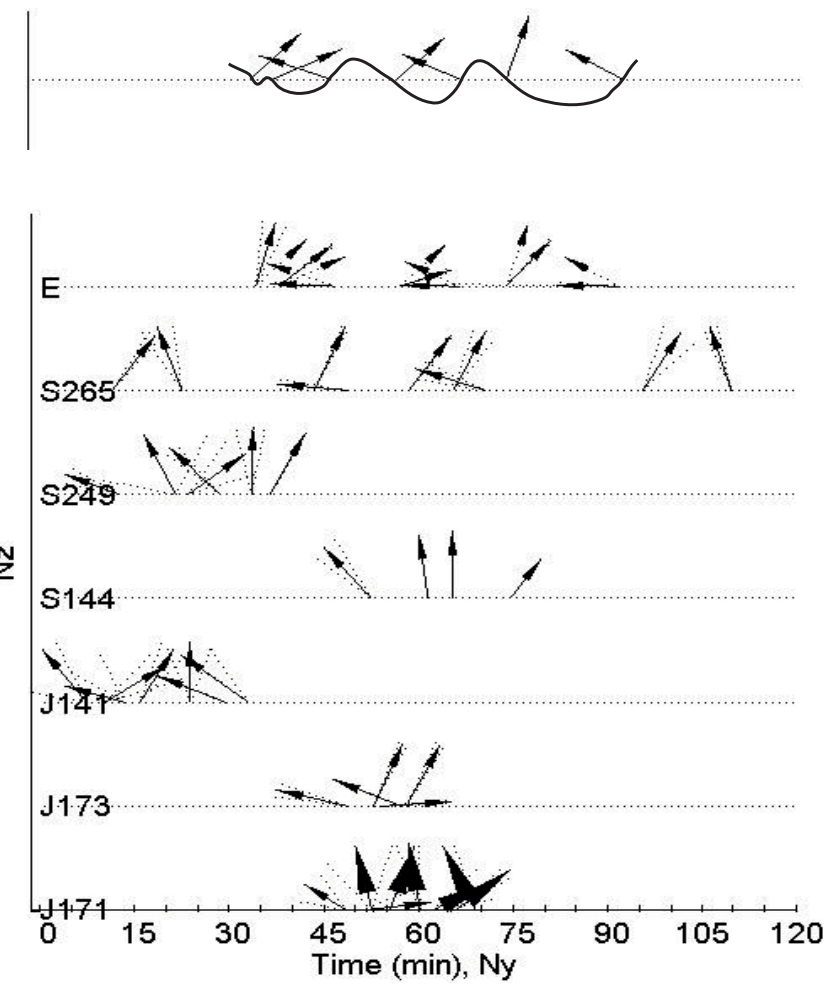

Fig. 4. Graphical representation of the current sheet normals y- and z-components for the Earth, Saturn DOY 249, 334 and 144 and Jupiter DOY 141, 173, 171. The top panel shows the timing normals for the Earth with superposed an artist's impression of the shape of the current sheet deduced from the normals. The bottom panel shows for the Earth both the timing (dotted) and MVA normals are plotted. The dashed lines forming cones around the solid arrows are the maximum error cones for the normal direction as determined from MVA. The horizontal axis functions as both the $N_{\mathrm{y}}$ direction and the time axis.

nate system. This transformation has the following effect that $B_{\mathrm{r}} \approx-B_{\mathrm{x}}, B_{\theta} \approx-B_{\mathrm{Z}}$ and $B_{\phi} \approx-B_{\mathrm{y}}$ for DOY 249 and 265 , whereas for DOY 144 the transformation is slighly different 

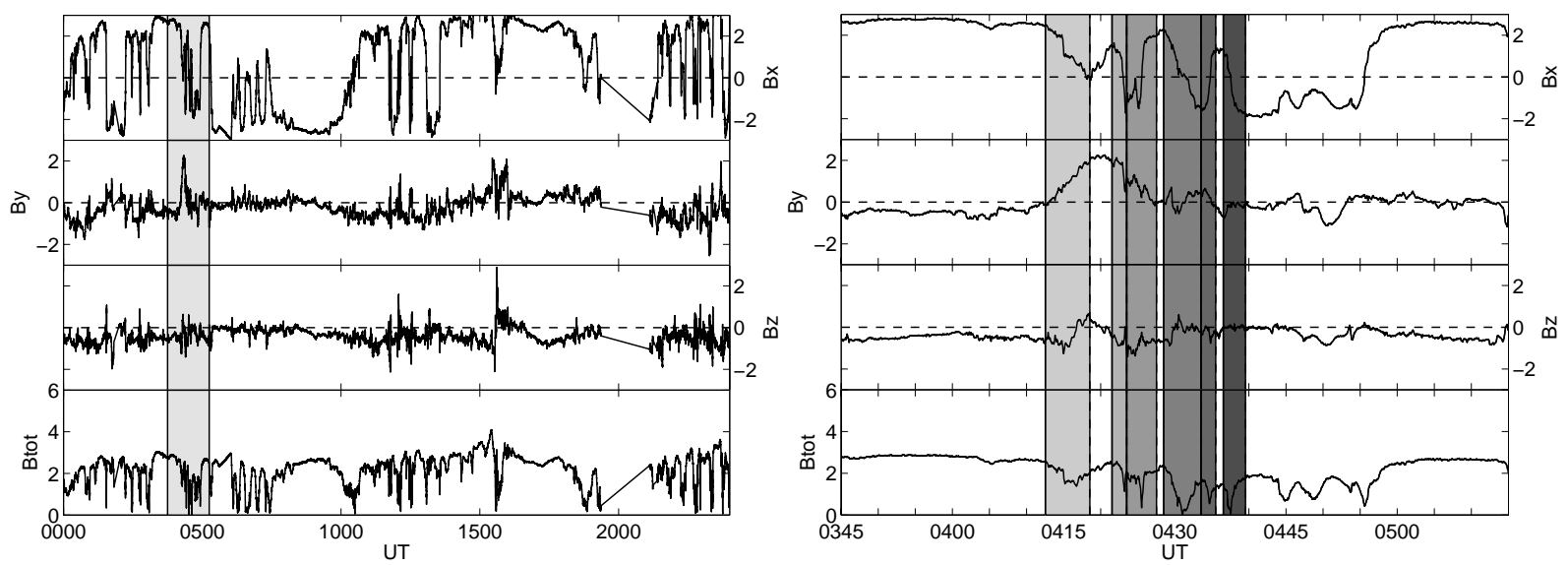

Fig. 5. Saturn flapping event on DOY 249. The left panel shows one full day of magnetic field data in KSM coordinates. The right panel shows a zoom in on the yellow shaded part in the left panel, showing the flapping event. The intervals for the MVA analysis are marked with filled areas of different shades of gray.

because of spacecraft location and $B_{\mathrm{r}} \approx B_{\mathrm{y}}, B_{\theta} \approx-B_{\mathrm{Z}}$ and $B_{\phi} \approx B_{\mathrm{x}}$.

\subsubsection{Saturn DOY 249}

The magnetic field data for DOY 249 (6 September 2006) and the interval 03:45-05:15 UT are shown in Fig. 5. It is clear that $B_{\mathrm{x}}$ crosses zero several times during the interval 04:06-04:42 UT. We define six intervals of strongly changing $B_{\mathrm{x}}$, where the last five are alternating decreases and increases of $B_{\mathrm{X}}$ with zero crossings. The intervals are marked with filled areas of different shades of gray in Fig. 5. Minimum variance analysis has been performed on these intervals and the results are given in Table 2 and graphically in Fig. 4. The quasi-period for this oscillation is $\sim 5 \mathrm{~min}$.

The results of the MVA show that the x-component of each minimum variance direction is relatively small compared to the $\mathrm{y}$ - or $\mathrm{z}$-component and is always positive. The y-component was always negative, but the z-component switched signs on each crossing between the second and fourth crossings. This is consistent with the observations of flapping current sheets in the Earth's magnetotail as presented by Sergeev et al. (2004) and in the Earth example above. The normal directions in the YZ-plane are shown in Fig. 4. The eigenvalue ratio for each vector was above 6 and was for most of the events was above 100, indicating that the minimum variance direction was well defined. Based on the MVA results, this event will be considered as a detection of magnetotail flapping at a period of $T \sim 5 \mathrm{~min}$.

\subsubsection{Saturn DOY 144}

One special event happened on DOY 144 (24 May 2006) during the interval 06:30-08:00 UT. The data are shown in Fig. 6, and it is clear that during this event the main oscilla- tion of the magnetic field is in $B_{\mathrm{y}}$ instead of $B_{\mathrm{x}}$. Also, there is a significant constant $B_{\mathrm{z}} \approx-3 \mathrm{nT}$ during this interval.

The MVA performed on the four intervals, shown in Table 3 and graphically in Fig. 4 does not show the expected direction change of the normal, which would be in the $\mathrm{XZ}$-plane as the flapping seems to take place in the $B_{\mathrm{y}}$ component. This can have two reasons: (1) Only the first interval has a well defined $B_{\mathrm{x}}=0$ crossing; (2) there is a strong $B_{\mathrm{Z}}$ during this event. The quasi-period for this oscillation is $\sim 9$ min.

For this event there are plasma data available with the correct pointing of the spacecraft (i.e., corotational flow enters the plasma instrument), that are shown in Fig. 7. We have plotted the $V_{\mathrm{Z}}$ component of the ion velocity and the density for $\mathrm{H}^{+}, \mathrm{H}_{2}^{+}$and $\mathrm{W}^{+}$. As the flapping motion seems to be in the $B_{\mathrm{y}}$ component, we have also plotted $d B_{\mathrm{y}} / d t$ (in contrast to $d B_{\mathrm{x}} / d t$ in e.g., Sergeev et al., 2004). During the flapping period there seems to be some indication that at 06:57 UT for $d B_{\mathrm{y}} / d t<0$ one finds for $\mathrm{H}_{2}^{+}$a positive velocity, whereas at 07:10 UT for $d B_{\mathrm{y}} / d t>0$ one finds for $\mathrm{H}_{2}^{+}$a negative velocity, showing the up-and-down motion of the current sheet over the spacecraft. However, due to relatively low count rates the velocity estimate has a rather large error, with other estimates than shown in this paper giving $V_{\mathrm{Z}}=0 \pm 10 \mathrm{~km} \mathrm{~s}^{-1}$ for this event (R. Wilson, personal communication, 2012). This makes it difficult to assign the flapping label to this event, as the MVA result is negative and the plasma data are inconclusive.

\subsubsection{Saturn DOY 265}

We show the data for DOY 265 (22 September 2006) and a zoom in on interval 11:00-13:30 UT in Fig. 8. Again a minimum variance analysis is applied to the intervals indicated in the figure. The results for the normal direction are shown 
Table 2. MVA analysis for the intervals during the Saturn DOY 249 flapping event. The lines starting with $\dagger$ mean that there was no $B_{\mathrm{X}}=0$ crossing during the interval. The current sheet normal is given by the eigen vector of the smallest eigenvalue and is chosen such that the $\mathrm{x}-$ component is positive and the $\mathrm{y}$-component negative. For a flapping event the $\mathrm{z}$-component should alternate between a positive and negative value in alternating $B_{\mathrm{x}}=0$ crossings. Also the ratio of the minimum $\lambda_{1}$ and intermediate $\lambda_{2}$ eigenvalues is given and the error estimates. A symbol $>$ for $\lambda_{2} / \lambda_{1}$ means a greater than 100 ratio and a symbol $<$ in the error estimates means a value less than 0.001 .

\begin{tabular}{c|cc|cc}
\hline Time & MVA & $\lambda_{\text {med }} / \lambda_{\min }$ & $\Delta \phi_{12}$ & $\Delta \phi_{13}$ \\
\hline$\dagger 04: 12: 32-04: 15: 32$ & $(0.59,-0.74,0.30)$ & 11 & 1.7 & 9.2 \\
$04: 21: 32-04: 23: 31$ & $(0.16,-0.43,0.88)$ & $>$ & $<$ & 52 \\
$04: 23: 31-04: 27: 32$ & $(0.26,-0.76,-0.59)$ & 6 & 2.3 & 19 \\
$04: 28: 30-04: 33: 32$ & $(0.25,-0.68,0.69)$ & 20 & 1.1 & 5.7 \\
$04: 33: 32-04: 35: 32$ & $(0.05,-0.02,-0.98)$ & $>$ & $<$ & 11 \\
$04: 36: 32-04: 39: 32$ & $(0.04,-0.46,-0.88)$ & $>$ & $<$ & $<$ \\
\hline
\end{tabular}

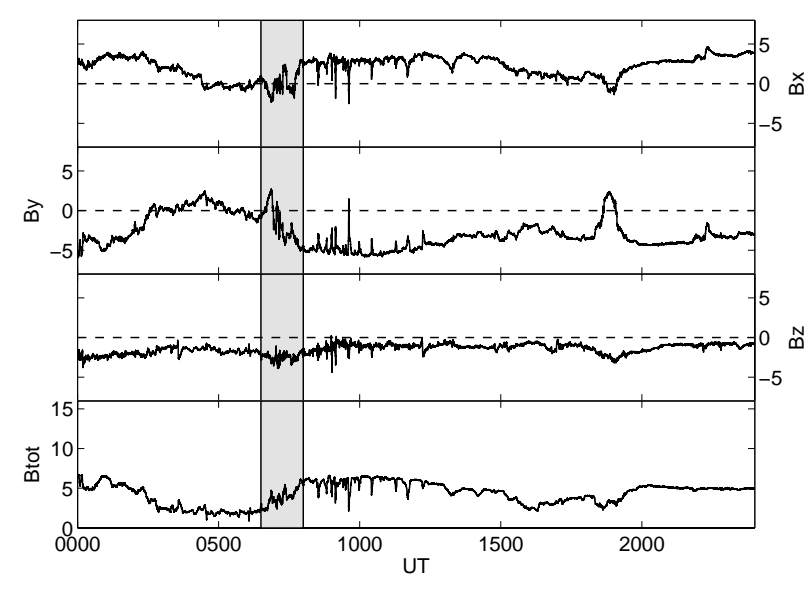

Fig. 6. Saturn flapping event on DOY 144. Format as in Fig. 5.

in Table 4 and graphically in Fig. 4. The quasi-period of this event was $\sim 15 \mathrm{~min}$.

Similar to the event on DOY 249 the minimum variance direction showed a pattern of small x-component, an alternatingly changing sign of the z-component and constant sign for the the $\mathrm{x}$ - and $\mathrm{y}$-components. The eigenvalue ratios were all above 4, suggesting the minimum variance direction was correct to within 20-30 degrees (Sergeev et al., 2006a, b), but were somewhat smaller than from DOY 249, with only two crossings with eigenvalue ratios greater than 200. Interestingly, there seems to be a skip at the sixth interval, which looks like a sharp change in the motion of the current sheet from the data shown in Fig. 8 right panel. There then is a rather large time gap, almost 1 period, before the next current sheet crossings and Cassini's ultimate crossing from the southern to the Northern Hemisphere. We will identify this event as magnetotail flapping.

\subsection{Observations at Jupiter}

At Jupiter, we use the Galileo magnetometer data (Kivelson et al., 1992) from the G8 orbit (i.e., the eighth orbit of Galileo around Jupiter, and the target flyby moon was Ganymede),

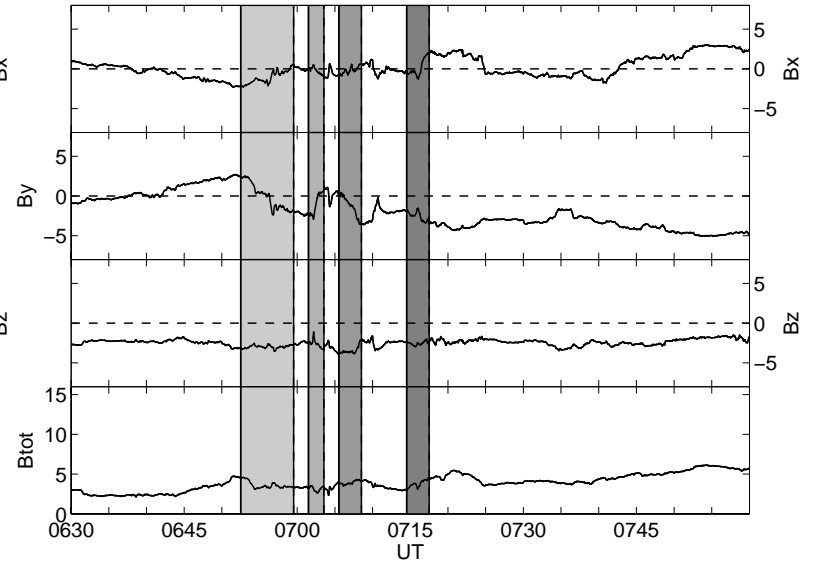

where the spacecraft is partly traversing the central part of the tail outbound from Jupiter and traversing the dusk-side tail during the inbound part of the orbit. Unfortunately, the plasma data available from this mission are too sparse to help with this investigation. The cruise data for the magnetic field were sampled at $24 \mathrm{~s}$ resolution.

We will discuss three events on DOY 141, 171 and 173 of 1997 in the following sections. The rotational (system III) period of Jupiter of 9 h 55' 29.71" (see e.g., Dessler, 1983) is apparent in the data. The tilted Jovian dipole $\left(\sim 9.6^{\circ}\right.$, see e.g., Bagenal et al., 2004) sweeps the current sheet up and down over the spacecraft in roughly $5 \mathrm{~h}$ intervals because Galileo's orbit remains near the Jovian equator. We searched these current sheet sweeps for flapping events.

\subsubsection{Jupiter DOY 141}

The magnetic field data for DOY 141 (21 May 1997) and for the interval 19:00-19:45 UT are shown in Fig. 9 in the Jovian-Solar-Magnetospheric (JSM) coordinate system, as defined above. The spacecraft is near the centre of the Jovian magnetotail. There is a low-amplitude oscillation of the magnetic field $B_{\mathrm{x}}$ during this current sheet crossing. We have 
Table 3. MVA analysis for the intervals during the Saturn DOY 144 flapping event. Format as in Table 2.

\begin{tabular}{c|cc|cc}
\hline Time & MVA & $\lambda_{\text {med }} / \lambda_{\min }$ & $\Delta \phi_{12}$ & $\Delta \phi_{13}$ \\
\hline $06: 52: 30-06: 59: 32$ & $(0.23,-0.63,0.74)$ & 7 & 5.7 & 11 \\
$07: 01: 30-07: 03: 32$ & $(0.35,-0.12,0.92)$ & $>$ & $<$ & $<$ \\
$07: 05: 31-07: 08: 31$ & $(0.19,-0.02,0.98)$ & $>$ & $<$ & $<$ \\
$\dagger 07: 14: 31-07: 17: 31$ & $(0.70,-0.41,-0.57)$ & $>$ & $<$ & $<$ \\
\hline
\end{tabular}

Table 4. MVA analysis for the intervals during the Saturn DOY 265 flapping event. Format as in Table 2.

\begin{tabular}{c|cc|cc}
\hline Time & MVA & $\lambda_{\text {med }} / \lambda_{\text {min }}$ & $\Delta \phi_{12}$ & $\Delta \phi_{13}$ \\
\hline$\dagger 11: 11: 30-11: 22: 31$ & $(0.20,0.57,-0.80)$ & 15 & 0.6 & 5.2 \\
$\dagger 11: 22: 31-11: 38: 31$ & $(0.18,0.33,0.93)$ & 4 & 1.7 & 12 \\
$\dagger 11: 43: 31-11: 48: 31$ & $(0.03,0.41,-0.92)$ & 240 & 1.1 & 2.3 \\
$\dagger 11: 48: 31-11: 56: 31$ & $(0.37,0.92,0.09)$ & 27 & 0.6 & 4.6 \\
$11: 58: 32-12: 05: 31$ & $(0.17,0.56,-0.80)$ & 240 & 0.6 & 1.7 \\
$12: 05: 31-12: 10: 31$ & $(0.26,0.43,-0.86)$ & 10 & 1.7 & 9.7 \\
$12: 10: 31-12: 17: 32$ & $(0.20,0.92,0.31)$ & 13 & 0.6 & 8.6 \\
$12: 35: 31-12: 45: 32$ & $(0.13,0.51,-0.85)$ & 5 & 0.6 & 14 \\
$12: 49: 32-13: 08: 32$ & $(0.11,0.29,0.94)$ & 9 & 1.7 & 5.7 \\
\hline
\end{tabular}

performed MVA analysis on the current sheet crossings, and the result is shown in Table 5 and graphically in Fig. 4.

The minimum variance directions show a similar pattern as for the Earth and Saturn, a small $x$-component, and an oscillating $\mathrm{z}$-component at constant sign of the $\mathrm{x}$ - and $\mathrm{y}$ component, indicative of a flapping event, a wave travelling from the centre of the magnetotail towards the flanks. Indeed, for the first five MVA directions we find this pattern, however, for the last two $B_{\mathrm{x}}=0$ crossings there seems to be a skip in the alternation. We note that the eigenvalue ratios for these MVAs were much lower than for the Saturn and Earth events, with only three events with an eigenvalue ratio greater than 4. However, based on the behaviour of the current sheet normal, which is as expected for a travelling wave, we identify this event as magnetotail flapping in the centre of the Jovian magnetotail. The quasi-period for this oscillation is $\sim 4 \mathrm{~min}$.

\subsubsection{Jupiter DOY 173}

The magnetic field data for DOY 173 (22 June 1997) and the interval 02:30-03:20 UT are shown in Fig. 10. There are several $B_{\mathrm{x}}=0$ crossings as well as $B_{\mathrm{y}}=0$. Galileo is located in the dawn flank of the Jovian magnetotail, where the magnetic field is influenced by flaring (see e.g., Huddleston et al., 1998; Belenkaya et al., 2005). The field lines are no longer mainly in the $\mathrm{x}$-direction, as they are near the centre of the magnetotail, but obtain a significant $y$-component. We, therefore, transform the data by rotating over $-30^{\circ}$ around the $\mathrm{z}$ axis, whereby the main variation of the magnetic field is in the rotated $X_{\mathrm{r}}$-component.
The MVA analysis is now performed on the rotated dataset and the results are shown in Table 6 and graphically in Fig. 4. The minimum variance directions for the $B_{\mathrm{Xr}}=0$ crossings are all very well defined, with all the eigenvalue ratios greater than 10. The directional pattern showing up in the current sheet normals shows a strong oscillation in amplitude between $Y_{\mathrm{r}}$ and $Z_{\mathrm{r}}$. The third crossing does not conform to this, with a negative $\mathrm{Z}$-component when we would expect a positive $\mathrm{z}$-component, however, this component was very small. There seems to be a skip in the last 2 crossings, which were not continuous with the first three, as can be seen in Fig. 10 (one $B_{\mathrm{Xr}}$-dip does not cross zero). The quasi-period of these oscillations is $\sim 3 \mathrm{~min}$.

\subsubsection{Jupiter DOY 171}

The data for DOY 171 (20 June 1997) and for the interval 14:30-15:30 UT are shown in Fig. 11. Again, there are multiple $B_{\mathrm{X}}=0$ crossings. However, there are also oscillations in the $B_{\mathrm{y}}$ component as in DOY 173 . As Galileo is in a similar region of the Jovian magnetotail as on DOY 173, we once more rotate the magnetic field data over $-30^{\circ}$ around the $\mathrm{z}$-axis to account for the flaring of the tail. We perform the MVA analysis on the rotated data and the results are shown in Table 7 and graphically in Fig. 4.

The current sheet normal directions show mostly a minimal $X_{\mathrm{r}}$-component, a sign-changing $\mathrm{z}$-component at constantly negative $X_{\mathrm{r}^{-}}$and $Y_{\mathrm{r}}$-components, which is indicative of a flapping event, where a travelling wave is moving in the $Y_{\mathrm{r}}$ direction. The eigenvalue ratios in this interval were comparable to but slightly higher than those from DOY 141. 
Table 5. MVA analysis for the intervals during the Jupiter DOY 141 flapping event. Format as in Table 2.

\begin{tabular}{c|cc|cc}
\hline Time & MVA & $\lambda_{\text {med }} / \lambda_{\min }$ & $\Delta \phi_{12}$ & $\Delta \phi_{13}$ \\
\hline$+19: 14: 31-19: 10: 15$ & $(-0.19,-0.60,0.78)$ & 1 & 2.3 & 63 \\
$+19: 10: 15-19: 13: 03$ & $(-0.21,-0.82,-0.53)$ & 4 & 2.9 & 15 \\
$19: 13: 27-19: 15: 27$ & $(-0.54,-0.80,0.24)$ & 3 & 1.7 & 49 \\
$1915: 51-19: 17: 51$ & $(-0.42,-0.46,-0.78)$ & 9 & 0.6 & 12 \\
$19: 23: 51-19: 26: 17$ & $(-0.49,-0.01,0.87)$ & 4 & 2.3 & 20 \\
$19: 29: 27-19: 31: 03$ & $(-0.14,-0.91,0.39)$ & $>$ & 0.2 & 0.3 \\
$19: 33: 03-19: 35: 27$ & $(-0.28,-0.64,-0.70)$ & 12 & 1.1 & 8.6 \\
\hline
\end{tabular}

Table 6. MVA for DOY Jupiter 173.

\begin{tabular}{c|cc|cc}
\hline Time & MVA & $\lambda_{\text {med }} / \lambda_{\min }$ & $\Delta \phi_{12}$ & $\Delta \phi_{13}$ \\
\hline $02: 48: 54-02: 50: 42$ & $(-0.08,-0.97,0.24)$ & 14 & 0.6 & 5.7 \\
$02: 52: 52-02: 53: 42$ & $(-0.16,-0.39,-0.91)$ & 120 & 0.6 & 2.9 \\
$02: 53: 53-02: 54: 40$ & $(-0.26,-0.96,-0.07)$ & $>$ & 0.6 & 1.1 \\
$02: 57: 18-02: 58: 05$ & $(-0.11,-0.91,0.39)$ & $>$ & $<$ & $<$ \\
$02: 58: 05-02: 59: 53$ & $(-0.01,-0.45,-0.90)$ & 32 & 0.6 & 4.0 \\
\hline
\end{tabular}

Except for the last two $B_{\mathrm{Xr}}=0$ crossings, where there is again a sign skip. The quasi-period for this event is $\sim 4 \mathrm{~min}$.

In summary: We examined the reliability of MVA to determine the true current sheet normal direction (taken to be that determined by multi-spacecraft timing analysis) for periods of flapping current sheets at Earth. Using this, we have studied several dynamic current sheet crossings at Saturn and Jupiter and determined the current sheet normal for multiple $B_{\mathrm{x}}=0$ crossings per event. For each planet the normals show strong oscillations, indicative of a "wavy twisted neutral sheet" as described by Zhang et al. (2002) (but see also Cowley, 1981). The period of the current sheet waves was 3-4 min at Jupiter and 5-15 min at Saturn, compared with 1-5 min at Earth (Sergeev et al., 2006a).

\section{Comparison with theory}

We have shown possible magnetotail flapping events for Jupiter and Saturn. We will now put these events into context with respect to the instability criteria discussed by Erkaev et al. (2008, 2009, 2010). Forsyth et al. (2009) showed recently that at Earth this model is preferred to describe the behaviour of the magnetotail during flapping. The so-called magnetic double gradient model follows from the linearised MHD equations and leads to a flapping frequency given by (SI versions taken from Forsyth et al., 2009):

$\omega_{\mathrm{f}}=\sqrt{\frac{1}{\mu_{0} \rho}\left\langle\frac{\partial B_{\mathrm{x}}}{\partial z}\right\rangle \frac{\partial B_{\mathrm{z}}}{\partial x}}$, where $\rho$ is the current sheet density and the dispersion relation is given by:

$\omega=\frac{\omega_{\mathrm{f}} k \Delta}{\sqrt{k^{2} \Delta^{2}+\lambda_{k}^{2}}}$,

where $\Delta$ is the current sheet half-thickness, $k$ is the wave number and $\lambda_{k}$ is the numerical solution to $\tan \lambda_{k}=k \Delta / \lambda_{k}$.

Unfortunately, there are no multi-spacecraft missions at Jupiter and Saturn, unlike at the Earth with the Cluster mission, that can deliver the instantaneous half-thickness $\Delta$ of the current sheet and the gradients of the magnetic field that are needed in Eqs. (7) and (8). However, there have been efforts to estimate the current sheet thickness for several Cassini revolutions by Sergis et al. (2011) and for Jupiter by Khurana and Kivelson (1989) and Khurana and Schwarzl (2005). As these are not simultaneous measurements with the events discussed in this paper, we will use magnetic field models that have shown to work well with the observations by spacecraft to describe the generic magnetotails of the giant planets and, therefore, predict the period of the flapping oscillations.

\subsection{Saturn}

The models for Saturn's magnetospheric field are based on the ring current model by Connerney et al. (1981, known as CAN81), which was originally developed for the Jovian magnetosphere. Using Cassini magnetometer data, this model was modified by Arridge et al. (2008) to include the seasonally dependent bowl-shape of the current sheet, which e.g., gives an explanation for the observed periodicities, which would not be expected in the almost perfectly aligned Kronian magnetic dipole magnetosphere. Also, they 
Table 7. MVA for Jupiter DOY 171. lines in italics mean no $B_{\mathrm{X}}=0$ crossing.

\begin{tabular}{c|cc|cc}
\hline Time & MVA & $\lambda_{\text {med }} / \lambda_{\min }$ & $\Delta \phi_{12}$ & $\Delta \phi_{13}$ \\
\hline 09:48:36-09:50:00 & $(-0.72,-0.57,0.38)$ & 4 & 4.6 & 22 \\
09:50:00-09:52:12 & $(-0.35,-0.93,-0.11)$ & 6 & 1.7 & 17 \\
09:52:12-09:55:26 & $(-0.24,-0.22,0.94)$ & 3 & 7.4 & 29 \\
09:55:26-09:57:00 & $(-0.14,-0.33,-0.93)$ & $>$ & $<$ & $<$ \\
10:00:14-10:02:13 & $(-0.11,-0.15,0.98)$ & 11 & 1.1 & 9.7 \\
10:02:13-10:04:12 & $(-0.47,-0.77,-0.41)$ & 6 & 1.1 & 17 \\
10:05:49-10:07:26 & $(-0.34,-0.73,-0.59)$ & 30 & 1.1 & 6.3 \\
10:09:00-10:11:49 & $(-0.10,-0.34,0.93)$ & 15 & 1.1 & 3.4 \\
\hline
\end{tabular}

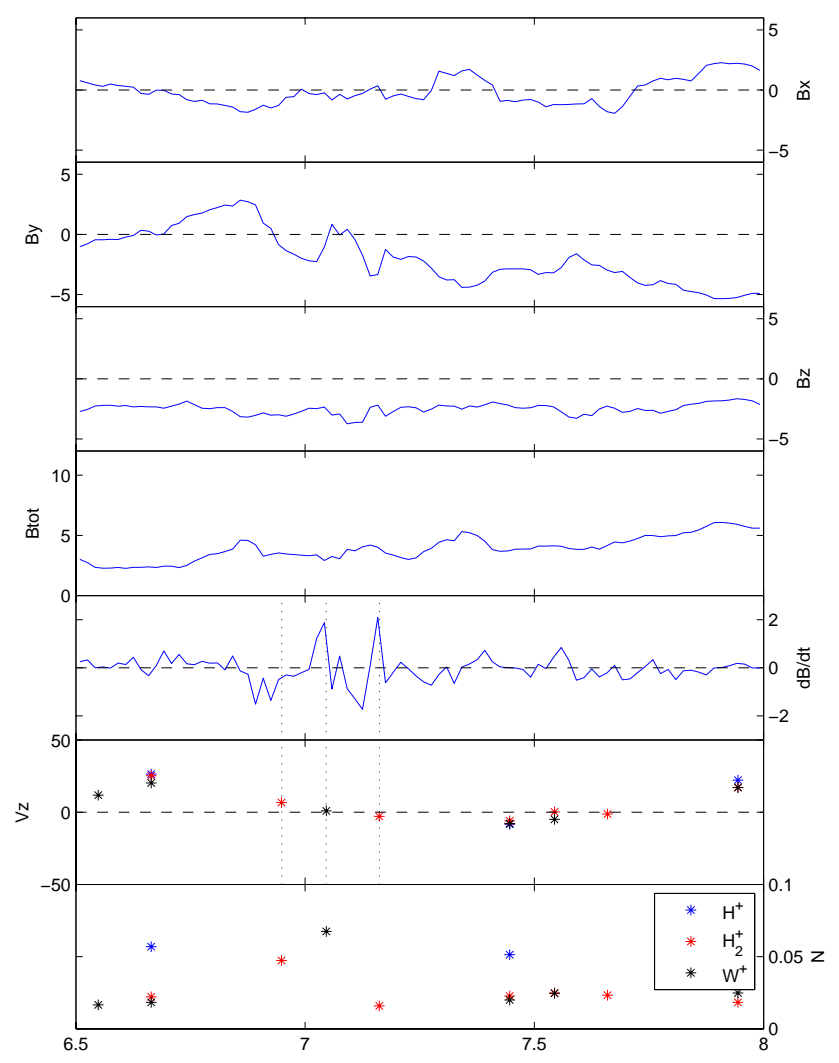

Fig. 7. Saturn flapping event on DOY 144. Shown are the magnetic field components in KSM coordinates and the total magnetic field. Then the time derivative $d B_{\mathrm{y}} / d t$, the z-component of the plasma velocity and the density for $\mathrm{H}^{+}$(blue), $\mathrm{H}_{2}^{+}$(red) and watergroup ions $\mathrm{W}^{+}$(black). The three vertical dotted lines show to which $d B_{\mathrm{y}} / d t$ the plasma $V_{\mathrm{Z}}$ map.

modified the CAN81 model to take into account the hinging of the magnetotail. This leads to a rather complicated model of the magnetic field equations, which describe the observed magnetic field well, but may be too detailed for the estimate of the flapping frequencies.

However, it has been shown by Dougherty et al. (2005) and Bunce et al. (2007) that the simple CAN81 model can rather well describe the magnetic field of the current sheet after the internal Kronian field has been subtracted from the data. Therefore, we will use the CAN81 model to calculate the flapping frequency as given by Eq. (7). As the flapping events at Saturn are taking place at $X_{\mathrm{KSM}}<-16 R_{\mathrm{S}}$, we use region III of the ring current model, which has the following equations for the magnetic field:

$$
\begin{aligned}
& B_{\mathrm{r}}^{\mathrm{III}}= \\
& \quad\left(\frac{\mu_{0} I_{0}}{2}\right)\left[\frac{1}{\rho}\left(F_{1}-F_{2}+2 D\right)-\frac{a^{2} r}{4}\left(\frac{1}{F_{1}^{3}}-\frac{1}{F_{2}^{3}}\right)\right], \\
& B_{\mathrm{z}}^{\mathrm{III}}= \\
& \quad\left(\frac{\mu_{0} I_{0}}{2}\right)\left[2 D\left(z^{2}+r^{2}\right)^{1 / 2}-\frac{a^{2}}{4}\left(\frac{z-D}{F_{1}^{3}}-\frac{z+D}{F_{2}^{3}}\right)\right], \\
& F_{1}=\left[(z-D)^{2}+a^{2}\right]^{1 / 2}, \\
& F_{2}=\left[(z+D)^{2}+a^{2}\right]^{1 / 2},
\end{aligned}
$$

where, with the parameters determined by Bunce et al. (2007), $\mu_{0} I_{0} \approx 60.4 \mathrm{nT}$ is the ring current, $D \approx 3 R_{\mathrm{S}}$ is the half-thickness of the current sheet, $a \approx 15.5 R_{\mathrm{S}}$ is the inner radius of the region III.

As through using magnetotail models we can only get rough estimates of the flapping frequency for generic magnetotail models; for simplicity we will assume that the spacecraft is at the midnight meridian, we can set $B_{\mathrm{x}}=B_{\mathrm{r}}$ and find the derivatives that are needed in Eq. (7):

$$
\begin{aligned}
\frac{d B_{\mathrm{x}}}{d z}= & \frac{\mu_{0} I_{0}}{2} \times \\
& {\left[-\frac{a^{2}}{4}\left(\frac{z-D}{\left(\left(a^{2}+(z-D)^{2}\right)^{3 / 2}\right.}-\frac{z+D}{\left(\left(a^{2}+(z+D)^{2}\right)^{3 / 2}\right.}\right)\right.} \\
+ & \left.\frac{2 D}{\left(z^{2}+x^{2}\right)^{1 / 2}}\right] \\
\frac{d B_{\mathrm{z}}}{d x}= & \frac{\mu_{0} I_{0} D x}{\left(z^{2}+x^{2}\right)^{3 / 2}} .
\end{aligned}
$$

The variation of the internal magnetic field needs to be added to these derivatives. The density in Saturn's magnetosphere during the flapping events discussed above is low, see e.g., 

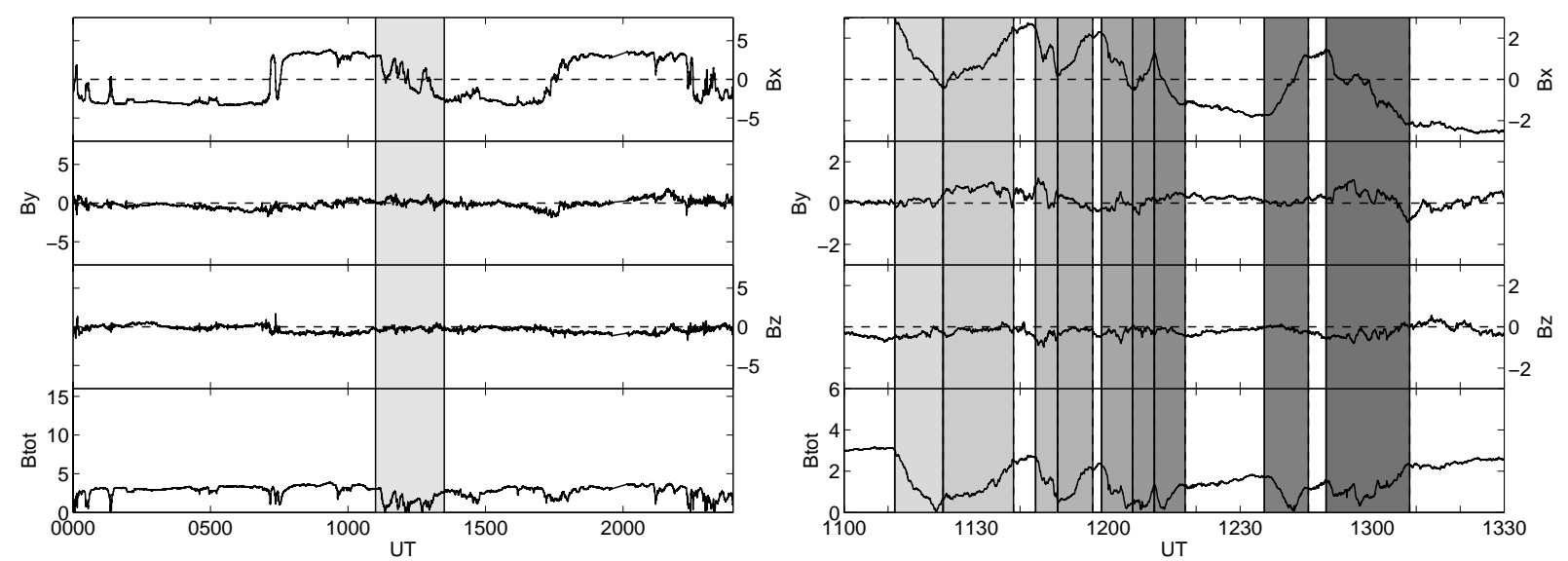

Fig. 8. Saturn flapping event on DOY 265. Format as in Fig. 5.
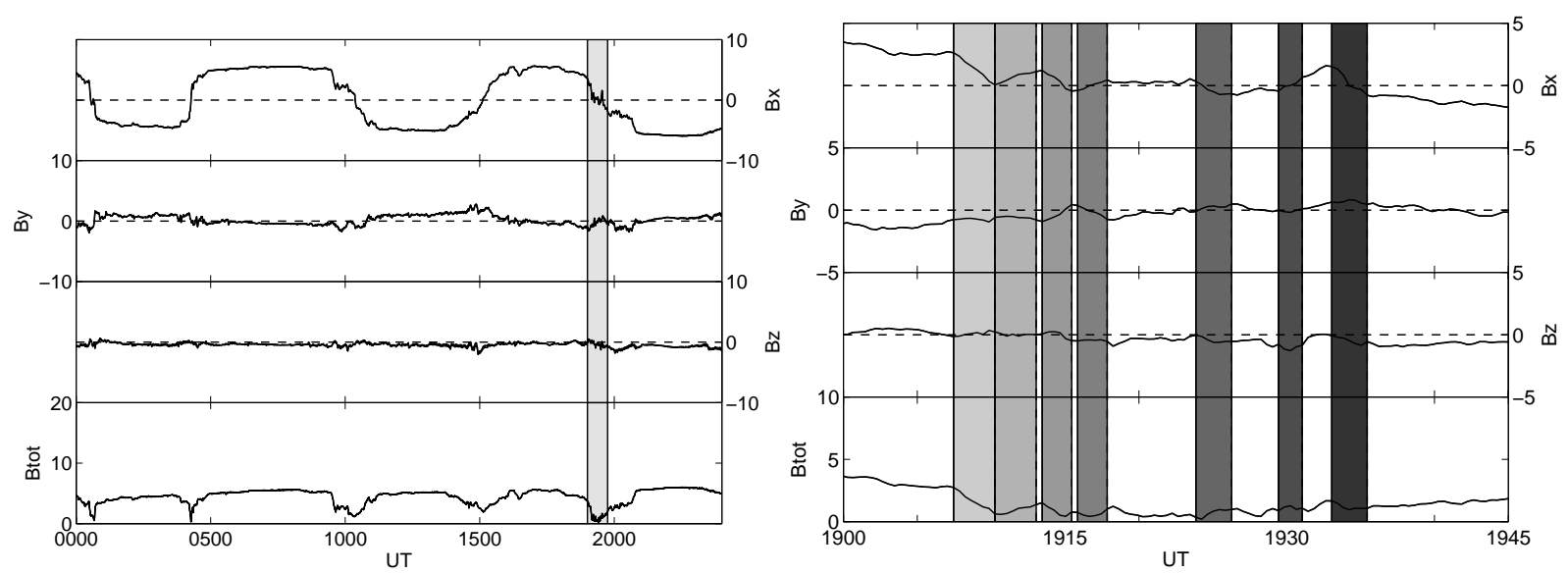

Fig. 9. Jupiter flapping event on DOY 141. The left panel shows one full day of magnetic field data in JSM coordinates. The right panel shows a zoom in on the yellow shaded part in the left panel, showing the flapping event. The intervals for the MVA analysis are marked with filled areas of different shades of gray.

Fig. 7, and we choose an upper limit $N \leq 0.1 \mathrm{~cm}^{-3}$ with an average mass of $\sim 10$ AMU. This leads to the following spread in the Kronian tail for $16 \leq X \leq 40$ to flapping periods of $15.5 \leq T \leq 49 \mathrm{~min}$. This range is at longer periods than measured above $(5-15 \mathrm{~min})$, however, this can well be due to the uncertainties put into this simple model, e.g., a current sheet thinning to half the nominal value, i.e., $D \Rightarrow 1.5 R_{\mathrm{S}}$ reduces the period by approximately a factor 1.5. The reason for reducing the half-thickness $\Delta$ is that the Earth magnetotail flapping is more likely to occur when the current sheet is thin (Sergeev et al., 2006b).

\subsection{Jupiter}

Khurana et al. (2004) describes several models for the Jovian magnetic field, and for the region which is interesting for this paper three models for the current sheet are compared: Connerney et al. (1981) (with a comment by Edwards et al., 2001), Goertz (1976) and Khurana (1997). The qualitative differences between the three models are shown in Fig. 24.12 in Khurana et al. (2004), and even though the model by Khurana (1997) seems to fit the Galileo data best, we opt here to use the model by Goertz (1976). The equations describing the magnetic field are much simpler to manipulate.

For the two important components of the magnetic field needed in Eq. (7) we find the following expressions for the field created by the current sheet:

$$
\begin{aligned}
& b_{\mathrm{r}}=\frac{b_{0}}{r^{a+1}} \tanh \left(\frac{z}{D(r)}\right), \\
& b_{\mathrm{z}}=\frac{a C B_{0} D(r)}{r^{a+2}},
\end{aligned}
$$

where $b_{0}=10^{-5} \mathrm{~T}, a=0.7$, the current sheet half-thickness can be estimated by $D(r)<4.6 / r^{0.3} R_{\mathrm{J}}$ for $r>2$ and for variations close to the equator $C \approx 10$ and both $r$ and $z$ are expressed in Jovian radii. To this, the dipole magnetic field $\boldsymbol{B}_{\mathrm{D}}$ needs to be added. The density of the current sheet is given by $N=5 \times 10^{7} r^{-6}$. 

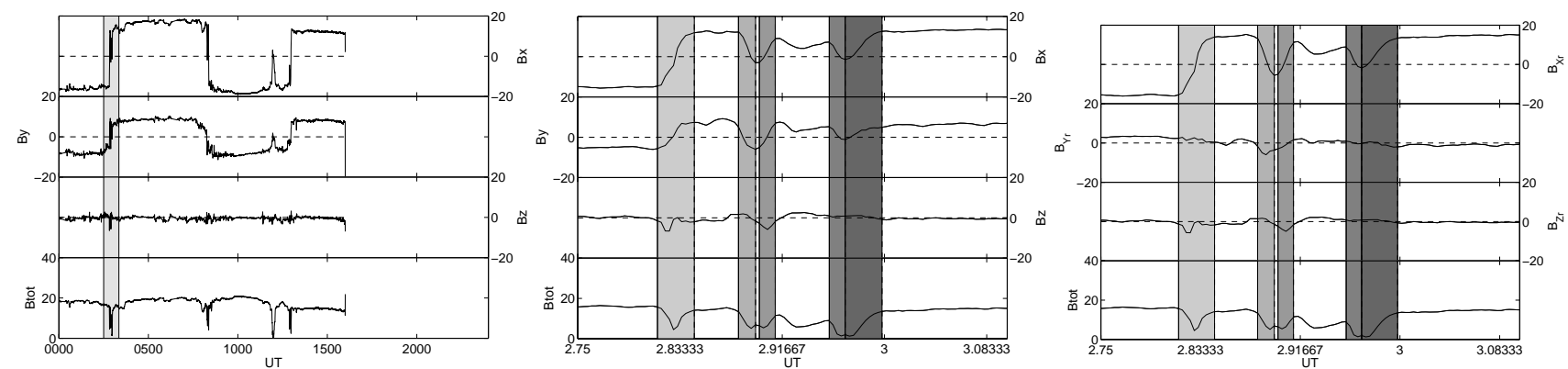

Fig. 10. Jupiter flapping event on DOY 173. Left and middle panel format as in Fig. 9. As the data need to be rotated to account for the magnetotail flaring, the right hand panel shows the data in the rotated system.
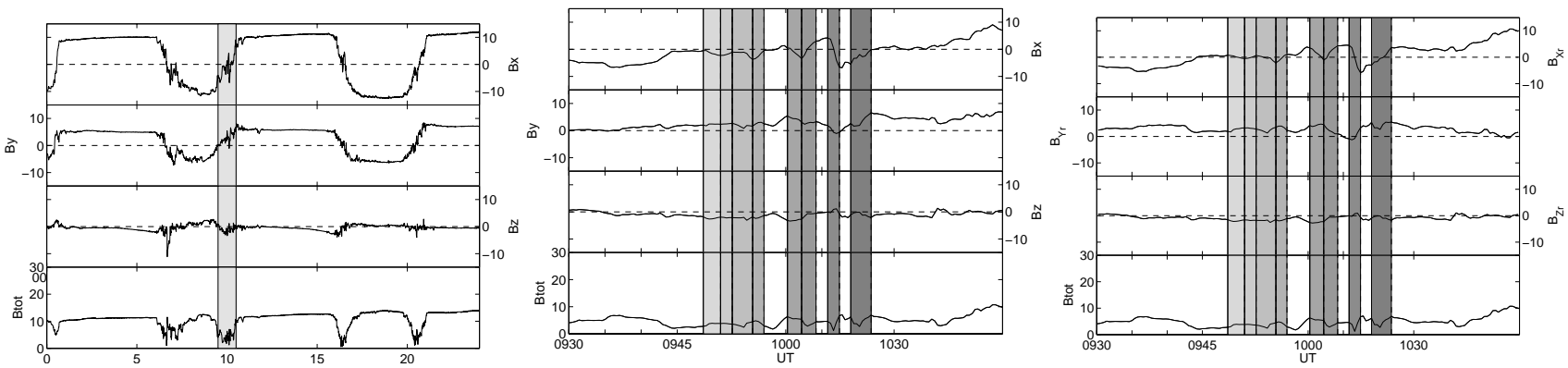

Fig. 11. Jupiter flapping event on DOY 171. Format as in Fig. 10.

There is no field-line bend-back in this magnetic field model (for that see e.g., Khurana, 1997), and for simplicity we assume that the spacecraft is at midnight local time, so we can say $b_{\mathrm{r}}=b_{\mathrm{x}}$ and we will replace $r$ with $x$. The derivatives for Eq. (7) are:

$$
\begin{aligned}
\frac{d B_{\mathrm{x}}}{d z} & =\frac{b_{0}}{x^{a+1}} \cosh ^{-2}\left(\frac{z}{D(x)}\right) D(x)^{-1}, \\
\frac{d B_{\mathrm{z}}}{d x} & =\frac{-3 B_{\mathrm{D}, \mathrm{z}}}{x^{4}}-4.6 a C b_{0}(a+2.3) x^{-(a+3.3)},
\end{aligned}
$$

where the $x$ variation in the dipole field is neglected. With Galileo located between $25 \leq x \leq 80 R_{\mathrm{J}}$, we find a flapping period for the Jovian tail of $21.5 \leq T_{\text {flap }} \leq 30.5 \mathrm{~min}$, which is higher than the quasi-periods found above (3-4 min). However, for the current sheet thickness the upper limit is used in this model, reducing that number to one third its value reduces the periods to half their values, closer to what is observed, but still off.

\section{Current sheet bifurcation}

As described in the introduction Israelevich and Ershkovich (2006); Israelevich et al. (2007) studied the bifurcation of the Jovian current sheet during intervals with multiple current sheet crossings observed by Voyager 2 and Galileo. Their conclusion was that a bifurcated current sheet happens only seldomly at Jupiter.
For the six events in this study we calculate the magnetic gradient. The magnetic field gradient is determined between consecutive points, i.e., at Galileo the $\Delta t=24 \mathrm{~s}$ and for Cassini $10 \mathrm{~s}$ (down-sampled from the $1 \mathrm{~s}$ resolution of the data in the Kronian magnetotail). The result for the different events are shown in Fig. 12, where the gradients are sorted with respect to the central $B_{\mathrm{X}}$ of the interval and binned with varying bin sizes per event.

For Jupiter DOY 141 and 171 there is no evidence for a bifurcated current sheet whereas for DOY 173 there is too sparse data to make any conclusion. DOY 141 shows a maximum around $B_{\mathrm{x}}=0$, with another maximum around $B_{\mathrm{X}} \approx$ 1 which comes from the first part of the interval (19:0019:15 UT). DOY 171 shows an almost constant value for $d B_{\mathrm{x}} / d t$, with no indication of bifurcation. For DOY 173 there are too few points in the bins to obtain any information about the structure of the current sheet. This is in agreement with the observations by Israelevich et al. (2007) that bifurcation is seldomly observed in the Jovian tail.

For Saturn DOY 265 there seems little evidence for an actual bifurcated current sheet, with a rather flat curve of $d B_{\mathrm{x}} / d t$. For DOY 144 the behaviour is slightly different, with elevated gradients around $B_{\mathrm{X}} \approx \pm 1 \mathrm{nT}$ with respect to $B_{\mathrm{X}}=0$. However, the error bars are rather large.

On DOY 249 there are two peaks in $d B_{\mathrm{x}} / d t$ at $B_{\mathrm{x}} \approx$ $\pm 0.5 \mathrm{nT}$, where the negative peak is well determined, whereas the positive peak had large error bars. This may well be a bifurcated current sheet, however, through the large $B_{\mathrm{y}}$ 

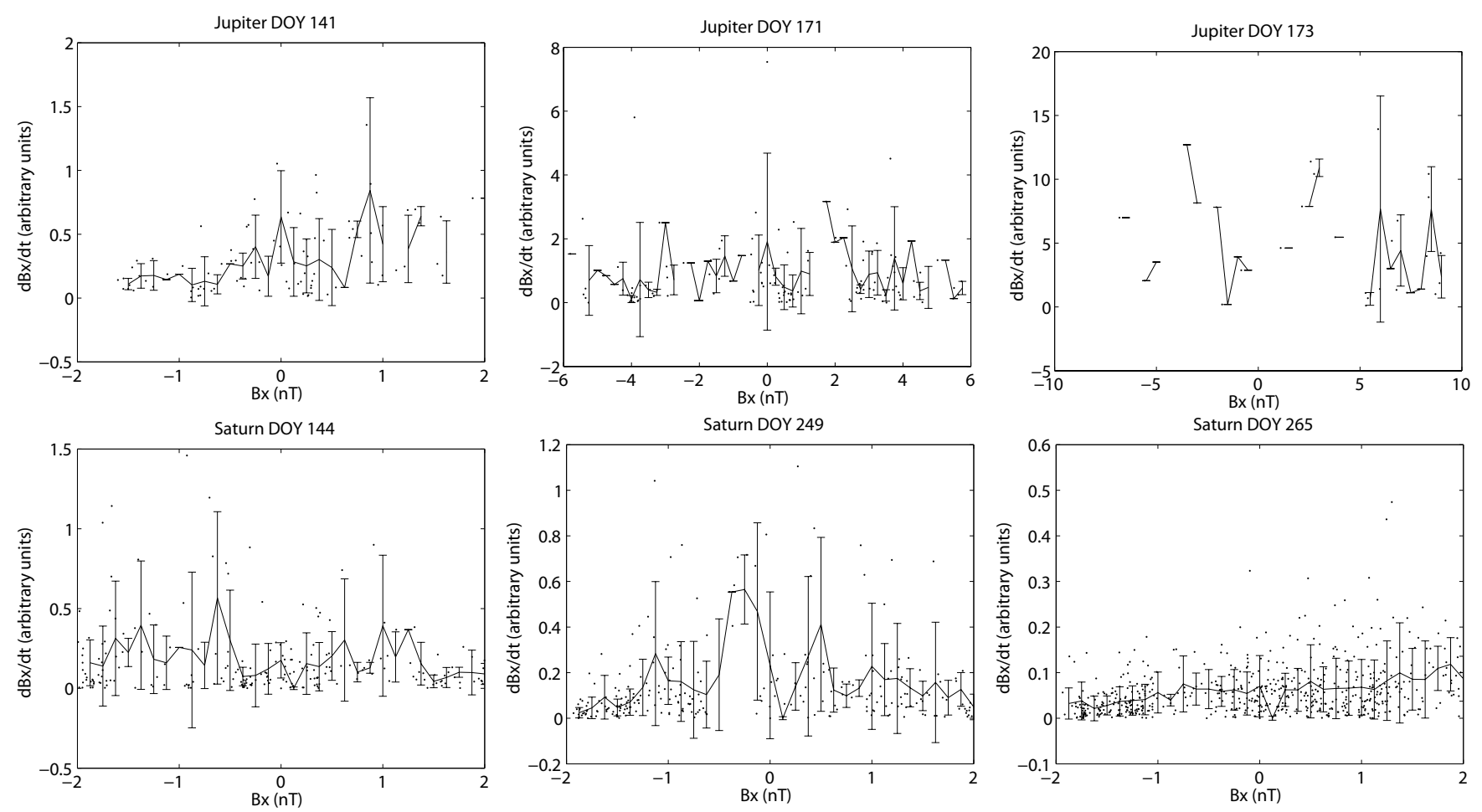

Fig. 12. Magnetic field gradients $d B_{\mathrm{X}} / d t$ as a function of $B_{\mathrm{X}}$. For each event the axes have a different ranges and the data are binned differently. Bin sizes: Jupiter DOY 141 - 0.125 nT; Jupiter DOY $171-0.25$ nT; Jupiter DOY 173 - 0.5 nT; Saturn DOY 144 - 0.125 nT; Saturn DOY 249 - 0.125 nT; Saturn DOY $265-0.125$ nT.

component between $\sim 04: 15$ and 04:30 UT the gradient determination may be contaminated. Taking out this interval does not improve the result significantly.

\section{Discussion}

We have performed a first comparative study of magnetotail flapping, comparing the details of the flapping process that were found in the Earth's magnetotail to the giant planets Jupiter and Saturn. Several events where there are multiple $B_{\mathrm{X}}=0$ crossings and/or oscillations of $B_{\mathrm{x}}$ whilst the spacecraft moves from one hemisphere of the tail to the other are studied using minimum variance analysis to obtain the current sheet normal.

At Earth it has been shown that some of these events are so-called flapping events, where a kink-mode like wave in the magnetotail moves from the centre to the flanks at a slight angle with the midnight meridian. The propagation direction can be determined for the Earth through the multi-spacecraft mission Cluster in combination with the Double Star mission.

Unfortunately, at Jupiter and Saturn there are no multispacecraft missions and, thus, we have to identify the possible flapping motion through different methods. We have chosen to look at the current sheet normal during the various crossings of the neutral sheet $B_{\mathrm{x}}=0$. In case of a wave moving as mentioned above, the normal is expected to flipflop in the YZ-plane and have only a minor component in $X$. In case that plasma data are available the up-and-down velocity of the magnetotail can be determined, which should have a correlation with the time gradient of the magnetic field. Unfortunately, for various reasons plasma data for these six events down the tails of the giant planets that can be used for this kind of analysis are scarce. For three possible flapping events selected at Cassini only for one event the pointing of the spacecraft was adequate to obtain a reliable estimate of the $V_{\mathrm{Z}}$ component. Thus, the main method for flapping identification was the current sheet normal direction.

We have shown two events at Saturn that could be identified as flapping based on the current sheet normal direction (DOY 249 and 265) and one event where the MVA analysis was inconclusive, but the available plasma data is hard pressed to support the interpretation that the Kronian magnetotail was flapping. Therefore, DOY 144 at Saturn cannot be labelled a magnetotail flapping event.

At Jupiter three events were shown where the MVA analysis clearly showed the motion of the current sheet normal in agreement with the expectations for flapping events. One thing that needs to be addressed is the rotation of the coordinate system for the flank events (DOY 171 and 173). The oscillation of the current sheet normal is "flapping-like" in the rotated coordinate system. This means that the waves 
travelling through the tail at a slight angle with respect to the $X_{\mathrm{JSM}}$-axis near the centre of the tail, propagate under a greater angle with respect to the $X_{\mathrm{JSM}_{\mathrm{S}}}$-axis near the flanks, but not with respect to the rotated $X_{\mathrm{r}}$-axis. This means that the waves move with the direction of the magnetic field in the tail.

Estimates for the period of the flapping were obtained from the magnetic field models for Saturn and Jupiter based on the double-gradient instability model. The estimated periods were above those observed, however, this can well be caused by errors in the input parameters for the magnetoplasma in both tails, like the current sheet thickness and the plasma density.

One of the differences between Jupiter, Saturn and the Earth is that the giant planets are rapid rotators, more than twice as fast as the Earth. This could have influence on the observed waves. At Earth a statistical study (Sergeev et al., 2006b) has investigated the occurrence frequency of flapping events as a function of $V_{\mathrm{Y}, \mathrm{GSM}}$, which shows that, although the distribution function is a narrow Gaussian, for relatively high velocities, $100 \leq\left|V_{\mathrm{Y}}\right| \leq 500 \mathrm{~km} \mathrm{~s}^{-1}$, there is a non-negligible number of events in their study. This range of values for $V_{\mathrm{Y}, \mathrm{GSM}}$ contains the corotational velocities at the giant planets. However, Sergeev et al. (2006b) do not show any data for events with strong $V_{\mathrm{y}}$, and do not indicate the wave period for these events. For the DOY 144 event at Saturn, the corotational velocity $V_{\phi} \approx 150 \mathrm{~km} \mathrm{~s}^{-1}$. Investigations by Runov et al. (2005) using multi-point Cluster measurements showed that the timing velocity of the kink-mode wave was equal to the bulk velocity of the plasma, indicating that the ion flow may transport the wave mode at relatively small velocities $V_{\mathrm{y}} \approx 25 \mathrm{~km} \mathrm{~s}^{-1}$. Higher bulk plasma flow velocity transporting the kink wave could possibly lead to Doppler shifting. This may be another reason why the estimated flapping periods from the generic magnetotail models are higher than what is measured by the spacecraft. Indeed, at dawn the propagation direction of the waves and the corotation velocity are in the same direction giving a Doppler blue shift.

At Earth it was shown that magnetotail flapping is related to storm or substorm activity in the magnetosphere (see e.g., Davey et al., 2012). We, therefore, compare the Jovian flapping events with the list of reconnection events presented by Vogt et al. (2010). It is found that the events of DOY 171 and 173 are during the same time interval at which reconnection was inferred, strengthening the case that in Jupiter magnetotail flapping is caused by the same kind of driver as at Earth.

There were only few flapping events in the datasets that were used in this study, for Saturn five possible events were found of which two (from three in this paper) could be clearly identified as flapping out of 157 tail crossings with multiple neutral sheet crossings. With a substorm occurrence rate of one per 2.4 days one would expect a substorm for every 5 neutral sheet crossings, which, however, need not be detected by the spacecraft if the flow channels are of limited width as at Earth (see e.g., Nakamura et al., 2004). Also, the predisposition of the current sheet for the "double gradient instability" may not occur as often in the Kronian magnetosphere. Similar comments can be made for the Jovian magnetotail.

A question can arise whether a bifurcated current sheet can produce a flapping signature when the spacecraft passes through. A crossing of a bifurcated current sheet would give rise to a signature in which $B_{\mathrm{X}}$ decreases, then increases again and then decreases, however, it will not necessarily lead to $B_{\mathrm{x}}=0$ crossings depending on the orbit of the spacecraft (see e.g., Runov et al., 2003, 2004), which were a requirement for the search of flapping events. Naturally, it cannot be excluded that current sheet bifurcation can play a part in the signatures that are measured, however, in this paper the study of possible bifurcation of the current sheets at Jupiter and Saturn showed that for only one event a case could be made.

Further statistical studies of magnetotail flapping at Jupiter and Saturn, using Galileo and Cassini data are planned to be performed in the near future. Some of the topics that cannot be addressed in a case study like this one are: Is magnetotail flapping dependent on local time; What is driving magnetotail flapping (one presented case shows a connection to magnetic reconnection); What is the occurrence rate of flapping events in comparison to that of magnetic reconnection. Some of these questions should be addressed before the arrival of Juno and JUICE at Jupiter, which then can deliver more observations.

Acknowledgements. Part of the work was done for ISSI working group number 195, "Investigating the Dynamics of Planetary Magnetotails", led by CMJ. MV would like to thank Hans Eichelberger for producing the Cluster and DoubleStar data, and Michelle Thomson and Rob Wilson for their help with the Cassini CAPS data. CMJ's work at UCL is supported by a Leverhulme Trust Early Career Fellowship and a Royal Astronomical Society Fellowship. AR is funded by the Belgian Fund for Scientific Research (FNRS). XJ was supported by NASA through grant NNX12AK34G and by the Cassini mission under contract JPL 1409449. APW holds an ESA Fellowship.

Topical Editor I. A. Daglis thanks four anonymous referees for their help in evaluating this paper.

\section{References}

Andrews, D. J., Cowley, S. W. H., M. K, D., Lamy, L., Provan, G., and Southwood, D. J.: Planetary period oscillations in Saturn's magnetosphere: Evolution of magnetic oscillations from southern summer to post-equinox, J. Geophys. Res., 117, A04224, doi:10.1029/2011JA017444, 2012.

Arridge, C. S., Achilleos, N., Dougherty, M. K., Khurana, K. K., and Russell, C. T.: Modeling the size and shape of Saturn's magnetopause with variable dynamic pressure, J. Geophys. Res., 111, A11227, doi:10.1029/2005JA011574, 2006.

Arridge, C. S., Khurana, K. K., Russell, C. T., Southwood, D. J., Achilleos, N., Dougherty, M. K., Coates, A. J., and 
Leinweber, H. K.: Warping of Saturn's magnetospheric and magnetotail current sheets, J. Geophys. Res., 113, a08217, doi:10.1029/2007JA012963, 2008.

Arridge, C. S., André, N., Khurana, K. K., Russell, C. T., Cowley, W. H., Provan, G., Andrews, D. J., Jackman, C. M., Coates, A. J., Sittler, E. C., Doughterty, M. K., and Young, D. T.: Periodic motion of Saturn's nightside plasma sheet, J. Geophys. Res., 116, A11205, doi:10.1029/2011JA016827, 2011.

Bagenal, F., Dowling, T. E., and McKinnon, W. B.: Jupiter: The planet, Satellites and Magnetosphere, Cambridge University Press, Cambridge, UK, 2004.

Balogh, A., Carr, C. M., Acuña, M. H., Dunlop, M. W., Beek, T. J., Brown, P., Fornacon, K.-H., Georgescu, E., Glassmeier, K.H., Harris, J., Musmann, G., Oddy, T., and Schwingenschuh, K.: The Cluster Magnetic Field Investigation: overview of in-flight performance and initial results, Ann. Geophys., 19, 1207-1217, doi:10.5194/angeo-19-1207-2001, 2001.

Belenkaya, E. S., Bobrovnikov, S. Y., Alexeev, I. I., Kalegaev, V. V., and Cowley, S. W. H.: A model of Jupiter's magnetospheric magnetic field with variable magnetopause flaring, Planet. Space Sci., 53, 863-872, 2005.

Bunce, E. J., Cowley, S. W. H., Alexeev, I. I., Arridge, C. S., Dougherty, M. K., Nichols, J. D., and Russell, C. T.: Cassini observations of the variation of Saturn's ring current parameters with system size, J. Geophys. Res., 112, A10202, doi:10.1029/2007JA012275, 2007.

Carr, C., Brown, P., Zhang, T. L., Gloag, J., Horbury, T., Lucek, E., Magnes, W., O’Brien, H., Oddy, T., Auster, U., Austin, P., Aydogar, O., Balogh, A., Baumjohann, W., Beek, T., Eichelberger, H., Fornacon, K.-H., Georgescu, E., Glassmeier, K.-H., Ludlam, M., Nakamura, R., and Richter, I.: The Double Star magnetic field investigation: instrument design, performance and highlights of the first year's observations, Ann. Geophys., 23, 2713-2732, doi:10.5194/angeo-23-2713-2005, 2005.

Connerney, J. E. P., Acuna, H. M., and Ness, N. F.: Modeling the jovian current sheet and inner magnetosphere, J. Geophys. Res., 87, 8370-8384, 1981.

Cowley, S. W. H.: Magnetospheric asymmetries associated with the y-component of the IMF, Planet. Space Sci., 29, 79-96, 1981.

Davey, E. A., Lester, M., Milan, S. E., and Fear, R. C.: Storm and substorm effects on magnetotail current sheet motion, J. Geophys. Res., 117, A02202, doi:10.1029/2011JA017112, 2012.

Dessler, A. J.: Physics of the Jovian Magnetosphere, Cambridge University Press, Cambridge, UK, 1983.

Dougherty, M. K., Kellock, S., Southwood, D. J., Balogh, A., Tsurutani, E. J. S. B. T., Gerlach, B., Glassmeier, K.-H., Gleim, F., Russell, C. T., Erdos, G., Neubauer, F. M., and Cowley, S. W. H.: The Cassini magnetic field investigation, Space Sci. Rev., 114, 331-383, doi:10.1007/s11214-004-1432-2, 2004.

Dougherty, M. K., Achilleos, N., André, N., Arridge, C. S., Balogh, A., Bertucci, C., Burton, M. E., Cowley, S. W. H., Erdos, G., Giampieri, G., Glassmeier, K.-H., Khurana, K. K., Leisner, J., Neubauer, F. M., Russell, C. T., Smith, E. J., Southwood, D. J., and Tsurutani, B. T.: Cassini magnetometer observations during Saturn orbit insertion, Science, 307, 1266-1270, doi:10.1126/science.1106098, 2005.

Edwards, T. M., Bunce, E. J., and Cowley, S. W. H.: A not on the vector potential of Connerney et al.'s model of the equatorial current sheet in Jupiter's magnetosphere, Planet. Space Sci., 49,
1115-1123, 2001.

Erkaev, N. V., Semenov, V. S., and Biernat, H. K.: Magnetic double gradient mechanism for flapping oscillations of a current sheet, Geophys. Res. Lett., 35, L02111, doi:10.1029/2007GL032277, 2008.

Erkaev, N. V., Semenov, V. S., Kubyshkin, I. V., Kubyshkina, M. V., and Biernat, H. K.: MHD model of the flapping motions in the magnetotail current sheet, J. Geophys. Res., 114, A03206, doi:10.1029/2008JA013728, 2009.

Erkaev, N. V., Semenov, V. S., and Biernat, H. K.: Hall magnetohydrodynamic effect for current sheet flapping oscillations related to the magnetic double gradient mechanism, Phys. Plasma, 17, 060703, doi:10.1063/1.3439687, 2010.

Fairfield, D. H.: Avarage and unusual locations of the Earth's magnetopause and bow shock, J. Geophys. Res., 76, 6700-6716, 1971.

Forsyth, C., Lester, M., Fear, R. C., Lucek, E., Dandouras, I., Fazakerley, A. N., Singer, H., and Yeoman, T. K.: Solar wind and substorm excitation of the wavy current sheet, Ann. Geophys., 27, 2457-2474, doi:10.5194/angeo-27-2457-2009, 2009.

Goertz, C. K.: The current sheet in Jupiter's magnetosphere, J. Geophys. Res., 81, 3368-3372, 1976.

Golovchanskaya, I. V. and Maltsev, Y. P.: On the identification of plasma sheet flapping waves observed by Cluster, Geophys. Res. Lett, 32, L02102, doi:10.1029/2004GL021552, 2005.

Harris, E. G.: On a plasma sheet separating regions of oppositely directed magnetic field, Nuovo Cimento, 23, 115-121, 1962.

Harvey, C. C.: Spatial gradients and volumetric tensor, in: Analysis Methods for Multi-Spacecraft Data, edited by: Paschmann, G. and Daly, P., pp. 307-322, ESA, Noordwijk, 1998.

Hesse, M. and Schindler, K.: Bifurcation of current sheets in plasmas, Phys. Fluids, 29, 2484-2492, 1986.

Hones, E. W., Baker, D. N., Bame, S. J., Feldman, W. C., Gosling, J. T., McComas, D. J., Zwickl, R. D., Slavin, J. A., Smith, E. J., and Tsurutani, B. T.: Structure of the magnetotail at $220 R_{\mathrm{E}}$ and its response to geomagnetic activity, J. Geophys. Res., 11, 5-7, 1984.

Huddleston, D. E., Russell, C. T., Kivelson, M. G., Khurana, K. K., and Bennett, L.: Location and shape of the Jovian magnetopause and bow shock, J. Geophys. Res., 103, 20075-20082, 1998.

Israelevich, P. L. and Ershkovich, A. I.: Bifurcation of Jovian magnetotail current sheet, Ann. Geophys., 24, 1479-1481, doi:10.5194/angeo-24-1479-2006, 2006.

Israelevich, P. L., Ershkovich, A. I., and Oran, R.: Bifurcation of the tail current sheet in Jovian magnetosphere, Planet. Space Sci., 55, 2261-2266, 2007.

Jackman, C. M., Russell, C. T., Southwood, D. J., Arridge, C. S., Achilleos, N., and Dougherty, M. K.: Strong rapid dipolarizations in Saturn's magnetotail: In situ evidence of reconnection, Geophys. Res. Lett., 34, L11203, doi:10.1029/2007GL029764, 2007.

Jackman, C. M., Arridge, C. S., Krupp, N., Bunce, E. J., Mitchell, D. G., McAndrews, H. J., Dougherty, M. K., Russell, C. T., Achilleos, N., Jones, G. H., and Coates, A. J.: A multi-instrument view of tail reconnection at Saturn, J. Geophys. Res., 113, A11213, doi:10.1029/2008JA013592, 2008.

Jackman, C. M., Arridge, C. S., McAndrews, H. J., Henderson, M. G., and Wilson, R. J.: Northward field excursions in Saturn's magnetotail and their relationship to mag- 
netospheric periodicities, Geophys. Res. Lett., 36, L16101, doi:10.1029/2009GL039149, 2009.

Jackman, C. M., Slavin, J. A., and Cowley, S. W. H.: Cassini observations of plasmoid structure and dynamics: Implications for the role of magnetic reconnection in magnetospheric circulation at Saturn, J. Geophys. Res., 116, A10212, doi:10.1029/2011JA016682, 2011.

Joy, S. P., Kivelson, M. G., Walker, R. J., Khurana, K. K., Russell, C. T., and Ogino, T.: Probabilistic models of the Jovian magnetopause and bow shock locations, J. Geophys. Res., 107, 1309, doi:10.1029/2001JA009146, 2002.

Khurana, K. K.: Euler potential models of Jupiter's magnetospheric field, J. Geophys. Res., 102, 11295-11306, 1997.

Khurana, K. K. and Kivelson, M. G.: On Jovian plasma sheet structure, J. Geophys. Res., 94, 11791-11803, 1989.

Khurana, K. K. and Schwarzl, H. K.: Global structure of Jupiter's magnetosspheric current sheet, J. Geophys. Res., 110, A07227, doi:10.1029/2004JA010757, 2005.

Khurana, K. K., Kivelson, M. G., Vasiliunas, V. M., Krupp, N., Woch, J., Lagg, A., Mauk, B. H., and Kurth, W. S.: The configuration of Jupiter's magnetosphere, in: Jupiter: The Planet, Satellites and Magnetosphere, edited by: Bagenal, F., Dowling, T. E., and McKinnon, W. B., pp. 593-616, Cambridge University Press, Cambridge, UK, 2004.

Kivelson, M. G., Khurana, K. K., Means, J. D., Russell, C. T., and Snare, R. C.: The Galileo magnetic field investigation, Space Sci. Rev., 60, 357-383, 1992.

Lui, A. T. Y., Meng, C.-I., and Akasofu, S.-I.: Wavy nature of the magnetotail neutral sheet, Geophys. Res. Lett., 5, 279-282, 1978.

Masters, A., Thomsen, M. F., Badman, S. V., Arridge, C. S., Young, D. T., Coates, A. J., and Dougherty, M. K.: Supercorotating return flow from reconnection in Saturn's magnetotail, Geophys. Res. Lett, 38, L03103, doi:10.1029/2010GL046149, 2011.

Nakamura, R., Baumjohann, W., Mouikis, C., Kistler, L. M., Runov, A., Volwerk, M., Asano, Y., Vörös, Z., Zhang, T. L., Klecker, B., Rème, H., and Balogh, A.: Spatial scale of high-speed flows in the plasma sheet observed by Cluster, Geophys. Res. Lett., 31, L09894, doi:10.1029/2004GL019558, 2004.

Provan, G., Andrews, D. J., Arridge, C. S., Coates, A. J., Cowley, S. W. H., Cox, G., Dougherty, M. K., and Jackman, C. M.: Dual periodicities in planetary-period magnetic field oscillations in Saturn's tail, J. Geophys. Res., 117, A01209, doi:10.1029/2011JA017104, 2012.

Runov, A., Nakamura, R., Baumjohann, W., Zhang, T. L., Volwerk, M., Eichelberger, H.-U., and Balogh, A.: Cluster observation of a bifurcated current sheet, Geophys. Res. Lett., 30, 1036, doi:10.1029/2002GL016136, 2003.

Runov, A., Sergeev, V., Nakamura, R., Baumjohann, W., Vörös, Z., Volwerk, M., Asano, Y., Klecker, B., Rème, H., and Balogh, A.: Properties of a bifurcated current sheet observed on $29 \mathrm{Au}-$ gust 2001, Ann. Geophys., 22, 2535-2540, doi:10.5194/angeo22-2535-2004, 2004.

Runov, A., Sergeev, V. A., Baumjohann, W., Nakamura, R., Apatenkov, S., Asano, Y., Volwerk, M., Vörös, Z., Zhang, T. L., Petrukovich, A., Balogh, A., Sauvaud, J.-A., Klecker, B., and Rème, H.: Electric current and magnetic field geometry in flapping magnetotail current sheets, Ann. Geophys., 23, 1391-1403, doi:10.5194/angeo-23-1391-2005, 2005.
Sergeev, V., Angelopulous, V., Carlson, C., and Sutcliffe, P.: Current sheet measurements within a flapping plasma sheet, J. Geophys. Res., 103, 9177-9188, 1998.

Sergeev, V., Runov, A., Baumjohann, W., Nakamura, R., Zhang, T. L., Volwerk, M., Balogh, A., Rème, H., Sauvaud, J.-A., André, M., and Klecker, B.: Current sheet flapping motion and structure observed by Cluster, Geophys. Res. Lett., 30, 1327, doi:10.1029/2002GL016500, 2003.

Sergeev, V., Runov, A., Baumjohann, W., Nakamura, R., Zhang, T. L., Balogh, A., Louarn, P., Sauvaud, J.-A., and Rème, H.: Orientation and propagation of current sheet oscillations, Geophys. Res. Lett., 31, L05807, doi:10.1029/2003GL019346, 2004.

Sergeev, V. A., Rounov, A., Baumjohann, W., Nakamura, R., Zhang, T. L., Apatenkov, S., Balogh, A., Rème, H., and Sauvaud, J.-A.: Cluster results on the magnetotail current sheet structure and dynamics, in: Proceedings Cluster and Double Star Symposium 5th Anniversary of Cluster in Space, edited by: Fletcher, K., p. P9.2, ESA SP, Noordwijk, 2006a.

Sergeev, V. A., Sormakov, D. A., Apatenkov, S. V., Baumjohann, W., Nakamura, R., Runov, A. V., Mukai, T., and Nagai, T.: Survey of large-amplitude flapping motions in the midtail current sheet, Ann. Geophys., 24, 2015-2024, doi:10.5194/angeo-242015-2006, 2006b.

Sergis, N., Arridge, C. S., Krimigis, S. M., Mitchell, D. G., Rymer, A. M., Hamilton, D. C., Krupp, N., Dougherty, M. K., and Coates, A. J.: Dynamics and seasonal variations in Saturn's magnetospheric plasma sheet, as measured by Cassini, J. Geophys. Res., 116, A04203, doi:10.1029/2010JA016180, 2011.

Sonnerup, B. and Cahill, L.: Magnetopause structure and attitude from Explorer 12 observations, J. Geophys. Res., 72, 171-183, 1967.

Sonnerup, B. U. Ö. and Scheible, M.: Minimum and maximum variance analysis, in: Analysis Methods for Multi-Spacecraft Data, edited by: Paschmann, G. and Daly, P., pp. 185-220, ESA, Noordwijk, 1998.

Speiser, T. W. and Ness, N. F.: The neutral sheet in the geomagnetic tail: Its motion, equivalent currents, and field line reconnection through it, J. Geophys. Res., 72, 131-141, 1967.

Toichi, T. and Miyazaki, T.: Flapping motions of the tail plasma sheet induced by the interplanetary magnetic field variations, Planet. Space. Sci., 24, 147-159, 1976.

Vogt, M. F., Kivelson, M. G., Khurana, K. K., Joy, S. P., and Walker, R. J.: Reconnection and flows in the Jovian magnetotail as inferred from magnetometer observations, J. Geophys. Res., 115, A06219, doi:10.1029/2009JA015098, 2010.

Volwerk, M., Zhang, T. L., Nakamura, R., Runov, A., Baumjohann, W., Glassmeier, K.-H., Takada, T., Eichelberger, H. U., Carr, C. M., Balogh, A., Klecker, B., and Rème, H.: Plasma flow channels with ULF waves observed by Cluster and Double Star, Ann. Geophys., 23, 2929-2935, doi:10.5194/angeo-23-2929-2005, 2005.

Young, D. T., Berthelier, J. J., Blanc, M., Burch, J. L., Coates, A. J., Goldstein, R., Hill, M. G. T. W., Johnson, R. E., Kelha, V., McComas, D. J., Sittler, E. C., Svenes, K. R., Szegö, K., Tanskanen, P., Ahola, K., Anderson, D., Bakshi, S., Baragiola, R. A., Barraclough, B. L., Black, R. K., Bolton, S., Booker, T., Bowman, R., Casey, P., Crary, F. J., Delapp, D., Dirks, G., Eaker, N., Funsten, H., Furman, J. D., Gosling, J. T., Hannula, H., Holmlund, C., Huomo, H., Illiano, J. M., Jensen, P., Johnson, M. A., Linder, D. R., Luntama, T., Maurice, S., McCabe, K. P., Mursula, K., 
Narheim, B. T., Nordholt, J. E., Preece, A., Rudzki, J., Ruitberg, A., Smith, K., Szalai, S., Thomsen, M. F., Viherkanto, K., Vilppola, J., Vollmer, T., Wahl, T. E., Wüest, M., Ylikorpi, T., and Zinsmeyer, C.: Cassini plasma spectrometer investigation, Space Sci. Rev., 114, 1-112, doi:10.1007/s11214-004-1406-4, 2004.

Zhang, T. L., Baumjohann, W., Nakamura, R., Balogh, A., and Glassmeier, K.-H.: A wavy twisted neutral sheet observed by Cluster, Geophys. Res. Lett., 29, 1899, doi:10.1029/2002GL015544, 2002.
Zhang, T. L., Nakamura, R., Volwerk, M., Runov, A., Baumjohann, W., Eichelberger, H. U., Carr, C., Balogh, A., Sergeev, V., Shi, J. K., and Fornacon, K.-H.: Double Star/Cluster observation of neutral sheet oscillations on 5 August 2004, Ann. Geophys., 23, 2909-2914, doi:10.5194/angeo-23-2909-2005, 2005.

Zhang, T. L., Baumjohann, W., Nakamura, R., Runov, A., Volwerk, M., Asano, Y., Vörös, Z., Eichelberger, H.-U., Sergeev, V., Shi, J. K., and Balogh, A.: A statistical survey of the magnetotail current sheet, Adv. Space Res., 38, 1834-1837, 2006. 